\title{
Convergence analysis for the equilibrium problems with numerical results
}

\author{
Sarawut Suwannaut and Atid Kangtunyakarn*
}

"Correspondence:

beawrock@hotmail.com

Department of Mathematics,

Faculty of Science, King Mongkut's

Institute of Technology Ladkrabang,

Bangkok 10520, Thailand

\begin{abstract}
In this paper, we propose an iterative scheme modified from the work of Ceng et al. (Nonlinear Anal. Hybrid Syst. 4:743-754, 2010) and Plubtieng and Punpaeng (J. Math. Anal. Appl. 336(1):455-469, 2007) to prove the strong convergence theorem for approximating a common element of the set of fixed points of nonspreading mappings and a finite family of the set of solutions of the equilibrium problem. Using this result, we obtain the strong convergence theorem for a finite family of nonspreading mappings and a finite family of the set of solutions of equilibrium problem. Moreover, in order to compare numerical results between the combination of the equilibrium problem and the classical equilibrium problem, some examples are given in one- and two-dimensional spaces of real numbers.
\end{abstract}

Keywords: nonspreading mapping; quasi-nonexpansive mapping; the combination of equilibrium problem

\section{Introduction}

Throughout this paper, let $C$ be a nonempty closed convex subset of a real Hilbert space $H$ with the inner product $\langle\cdot, \cdot\rangle$ and the norm $\|\cdot\|$. We denote weak convergence and strong convergence by the notations ' $\rightarrow$ ' and ' $\rightarrow$ ', respectively. We use $\mathbb{R}$ to denote the set of real numbers and $\operatorname{Fix}(\mathcal{T})$ to represent the set of fixed points of $\mathcal{T}$, where $\mathcal{T}$ is a mapping from $C$ into itself.

In 2008, Kohsaka and Takahashi [1] introduced the nonspreading mapping $\mathcal{T}$ in Hilbert space $H$ as follows:

$$
2\|\mathcal{T} u-\mathcal{T} v\|^{2} \leq\|\mathcal{T} u-v\|^{2}+\|u-\mathcal{T} v\|^{2}, \quad \forall u, v \in C .
$$

In 2009, it was shown by Iemoto and Takahashi [2] that (1.1) is equivalent to the following equation:

$$
\|\mathcal{T} u-\mathcal{T} v\|^{2} \leq\|u-v\|^{2}+2\langle u-\mathcal{T} u, v-\mathcal{T} v\rangle, \quad \text { for all } u, v \in C \text {. }
$$

Many researchers proved the strong convergence theorem for a nonspreading mapping and some related mappings in Hilbert space; see for example [3-6].

@2014 Suwannaut and Kangtunyakarn; licensee Springer. This is an Open Access article distributed under the terms of the Creative Commons Attribution License (http://creativecommons.org/licenses/by/2.0), which permits unrestricted use, distribution, and reproduction in any medium, provided the original work is properly cited. 
Let $B: C \rightarrow H$. The variational inequality problem is to find a point $u \in C$ satisfying the following inequality:

$$
\langle B u, v-u\rangle \geq 0,
$$

for all $v \in C$. Moreover, $\operatorname{VI}(C, B)$ is used to denote the set of solutions of (1.2).

Let $\Phi: C \times C \rightarrow \mathbb{R}$ be a bifunction. The classical equilibrium problem for $\Phi$ is to find $u \in C$ satisfying the following inequality:

$$
\Phi(u, v) \geq 0, \quad \forall v \in C
$$

We use $\operatorname{EP}(\Phi)$ to represent the set of solution of (1.3).

Let the bifunction $\Phi$ satisfy the following conditions for solving the equilibrium problem.

(A1) $\Phi(u, u)=0$ for all $u \in C$;

(A2) $\Phi$ is monotone, i.e., $\Phi(u, v)+\Phi(v, u) \leq 0$ for all $u, v \in C$;

(A3) for each $u, v, w \in C$,

$$
\lim _{t \rightarrow 0^{+}} \Phi(t w+(1-t) u, v) \leq \Phi(u, v)
$$

(A4) for each $u \in C, v \mapsto \Phi(u, v)$ is convex and lower semicontinuous.

In 1994, Blum and Oettli [7] showed that the classical equilibrium problem (1.3) covers monotone inclusion problems, saddle point problems, variational inequality problems, minimization problems, Nash equilibria in noncooperative games, vector equilibrium problems, and certain fixed point problems.

Let $\Psi=\left\{\Phi_{i}\right\}_{i=1,2, \ldots, N}$ be a finite family of bifunctions from $C \times C$ to $\mathbb{R}$. The system of equilibrium problem for $\Psi$ is to determine common equilibrium points for $\Psi=\left\{\Phi_{i}\right\}_{i=1,2, \ldots, N}$, that is, the set

$$
\mathrm{EP}(\Psi)=\left\{u \in C: \Phi_{i}(u, v) \geq 0, \forall v \in C, \forall i \in 1,2, \ldots, N\right\} .
$$

The problem (1.4) extends (1.3) to a system of such problems covering various forms of feasibility problems [8]. Several iterative algorithms are proposed to solve the equilibrium problems and a finite family of equilibrium problems; see, for instance, [8-14].

Example 1.1 Let $\Psi=\left\{\Phi_{i}\right\}_{i=1,2, \ldots, N}$ be a finite family of bifunctions from $C \times C$ to $\mathbb{R}$, where the bifunctions $\Phi_{i}$ are defined by

$$
\Phi_{i}(u, v)=i(v-u)(v+2 u-3), \quad \text { for every } u, v \in \mathbb{R} .
$$

For each $i=1,2, \ldots, N$, it is obvious that the $\Phi_{i}(x, y)$ satisfy (A1)-(A4). Then we obtain

$$
\operatorname{EP}(\Psi)=\bigcap_{i=1}^{N} \operatorname{EP}\left(\Phi_{i}\right)=\{1\} .
$$

In 2010, Peng et al. [15] proposed the following iterative algorithm for solving a family of infinite nonexpansive mappings and a finite family of equilibrium problems in Hilbert 
space:

$$
\left\{\begin{array}{l}
z_{1}=z \in H, \\
u_{n}=T_{\beta_{n}}^{F_{m}} T_{\beta_{n}}^{F_{m-1}} \cdots T_{\beta_{n}}^{F_{2}} T_{\beta_{n}}^{F_{1}} z_{n}, \\
v_{n}=P_{C}\left(I-s_{n} A\right) u_{n}, \\
z_{n+1}=\alpha_{n} \gamma f\left(W_{n} z_{n}\right)+\left(I-\alpha_{n} B\right) W_{n} P_{C}\left(I-r_{n} A\right) v_{n}, \quad \forall n \in \mathbb{N} .
\end{array}\right.
$$

Under some appropriate conditions, they proved that $\left\{z_{n}\right\},\left\{v_{n}\right\}$, and $\left\{u_{n}\right\}$ converge strongly to $q=P_{\Omega}(\gamma f+(I-B))(q)$, where $\Omega=\bigcap_{i=1}^{\infty} \operatorname{Fix}\left(S_{i}\right) \cap \operatorname{VI}(C, A) \cap \bigcap_{k=1}^{m} \operatorname{EP}\left(F_{k}\right)$ and $f$ is a contractive mapping on $H$.

Over the past few years, many researchers have started working on the methods for finding a common solution of a finite family of equilibrium problems in Hilbert space; see, for instance, [16-18].

In 2013, Suwannaut and Kangtunyakarn [12] introduced the combination of equilibrium problem which is to find $u \in C$ such that

$$
\left(\sum_{i=1}^{N} a_{i} \Phi_{i}\right)(u, v) \geq 0, \quad \forall v \in C
$$

where $\Phi_{i}: C \times C \rightarrow \mathbb{R}$ are bifunctions and $a_{i} \in(0,1)$ with $\sum_{i=1}^{N} a_{i}=1$, for every $i=$ $1,2, \ldots, N$. The set of solutions (1.6) is denoted by $\operatorname{EP}\left(\sum_{i=1}^{N} a_{i} \Phi_{i}\right)$.

If $\Phi_{i}=\Phi$, for all $i=1,2, \ldots, N$, then the combination of equilibrium problem (1.6) reduces to the classical equilibrium problem (1.3).

Moreover, they obtain Lemma 2.10 as shown in the next section.

Example 1.2 For every $i=1,2,3$, let the bifunctions $\Phi_{i}: \mathbb{R} \times \mathbb{R} \rightarrow \mathbb{R}$, be given by

$$
\begin{aligned}
& \Phi_{1}(u, v)=(v-u)(v+u-2), \\
& \Phi_{2}(u, v)=(v-u)(3 v+5 u-8), \\
& \Phi_{3}(u, v)=(v-u)(9 v+12 u-21), \quad \forall u, v \in \mathbb{R} .
\end{aligned}
$$

For all $i=1,2,3$, it is obvious that the $\Phi_{i}(u, v)$ satisfy (A1)-(A4). Let $a_{1}=\frac{1}{2}, a_{2}=\frac{1}{9}$ and $a_{3}=\frac{7}{18}$, thus we have

$$
\sum_{i=1}^{3} a_{i} \Phi_{i}(u, v)=\frac{1}{18}(v-u)(78 v+103 u-181)
$$

This implies that

$$
\operatorname{EP}\left(\sum_{i=1}^{3} a_{i} \Phi_{i}\right)=\bigcap_{i=1}^{3} \operatorname{EP}\left(\Phi_{i}\right)=\{1\}
$$

Remark 1.3 For all $i=1,2, \ldots, N$, let the mapping $\mathcal{A}_{i}: C \rightarrow H$ be defined by $\Phi_{i}(u, v)=$ $\left\langle\mathcal{A}_{i} u, v-u\right\rangle$ for all $u, v \in C$. For each $i=1,2, \ldots, N$, if $\Phi_{i}(u, v)=\left\langle\mathcal{A}_{i} u, v-u\right\rangle \geq 0$ for all 
$u, v \in C$, and $i=1,2, \ldots, N$, then $\operatorname{EP}\left(\Phi_{i}\right)=\operatorname{VI}\left(C, \mathcal{A}_{i}\right)$. Hence we have

$$
\operatorname{EP}\left(\sum_{i=1}^{N} a_{i} \Phi_{i}\right)=\bigcap_{i=1}^{N} \operatorname{EP}\left(\Phi_{i}\right)=\bigcap_{i=1}^{N} \operatorname{VI}\left(C, \mathcal{A}_{i}\right) .
$$

After we have studied research related to equilibrium problems, we obtain the following question.

Question Is it possible to prove strong convergence theorem for a finite family of equilibrium problem using different method from the result of Peng et al. [15], Piri [17] and references therein?

Inspired and motivated by the work of Iemoto and Takahashi [2], Suwannaut and Kangtunyakarn [12] and related research, we propose an iterative scheme modified from the work of Plubtieng and Punpaeng [19] and Ceng et al. [11] to prove the strong convergence theorem for approximating a common element of the set of fixed points of a nonspreading mapping and a finite family of the set of solutions of equilibrium problems using Lemma 2.10 and a different method from the work of Peng et al. [15] and Piri [17] and references therein. Moreover, some examples are given in order to compare the numerical results between the combination of the equilibrium problem and the classical equilibrium problem.

\section{Preliminaries}

We now recall the following definition and well-known lemmas.

\section{Definition 2.1}

(i) $\mathcal{A}$ is strongly positive operator on $H$ if there exists a constant $\beta>0$ such that

$$
\langle\mathcal{A} u, u\rangle \geq \beta\|u\|^{2}, \quad \forall u \in H
$$

(ii) $\mathcal{T}$ is a nonexpansive mapping if

$$
\|\mathcal{T} u-\mathcal{T} v\| \leq\|u-v\|, \quad \forall u, v \in C
$$

(iii) For every $u \in H$, there is a unique nearest point $P_{C} u$ in $C$ such that

$$
\left\|u-P_{C} u\right\| \leq\|u-v\|, \quad \forall v \in C
$$

Such an operator $P_{C}$ is called the metric projection of $H$ onto $C$.

Lemma 2.1 ([20]) For a given $w \in H$ and $u \in C$,

$$
u=P_{C} w \quad \Leftrightarrow \quad\langle u-w, v-u\rangle \geq 0, \quad \forall v \in C .
$$

Furthermore, $P_{C}$ is a nonexpansive mapping. 
Lemma 2.2 ([21]) Each Hilbert space H satisfies Opial's condition, i.e., for any sequence $\left\{u_{n}\right\} \subset H$ with $u_{n} \rightarrow u$, the inequality

$$
\liminf _{n \rightarrow \infty}\left\|u_{n}-u\right\|<\liminf _{n \rightarrow \infty}\left\|u_{n}-v\right\|
$$

holds for every $v \in H$ with $v \neq u$.

Lemma 2.3 ([22]) Let $\left\{u_{n}\right\}$ be a sequence of nonnegative real numbers satisfying

$$
u_{n+1} \leq\left(1-\beta_{n}\right) u_{n}+\eta_{n}, \quad \forall n \geq 0,
$$

where $\alpha_{n}$ is a sequence in $(0,1)$ and $\left\{\eta_{n}\right\}$ is a sequence such that

(1) $\sum_{n=1}^{\infty} \beta_{n}=\infty$,

(2) $\lim \sup _{n \rightarrow \infty} \frac{\eta_{n}}{\beta_{n}} \leq 0$ or $\sum_{n=1}^{\infty}\left|\eta_{n}\right|<\infty$.

Then $\lim _{n \rightarrow \infty} u_{n}=0$.

Lemma 2.4 ([4]) Let $H$ be a real Hilbert space. Then the following results hold:

(i) For all $u, v \in H$ and $t \in[0,1]$,

$$
\|t u+(1-t) v\|^{2}=t\|u\|^{2}+(1-t)\|v\|^{2}-t(1-t)\|u-v\|^{2},
$$

(ii) $\|u+v\|^{2} \leq\|u\|^{2}+2\langle v, u+v\rangle$, for each $u, v \in H$.

Lemma 2.5 ([20]) Let $H$ be a Hilbert space, let $C$ be a nonempty closed convex subset of $H$ and let $\mathcal{A}$ be a mapping of $C$ into $H$. Then, for $\alpha>0$,

$$
\operatorname{Fix}\left(P_{C}(I-\alpha \mathcal{A})\right)=\operatorname{VI}(C, \mathcal{A})
$$

where $P_{C}$ is the metric projection of $H$ onto $C$.

Lemma 2.6 ([23]) Assume $\mathcal{A}$ is a strongly positive linear bounded operator on a Hilbert space $H$ with coefficient $\beta>0$ and $0<\delta<\|\mathcal{A}\|^{-1}$. Then $\|I-\delta \mathcal{A}\| \leq 1-\delta \beta$.

Lemma 2.7 ([2]) Let $C$ be a nonempty closed convex subset of $H$. Then a mapping $\mathcal{T}: C \rightarrow$ $C$ is nonspreading if and only if

$$
\|\mathcal{T} u-\mathcal{T} v\|^{2} \leq\|u-v\|^{2}+2\langle u-\mathcal{T} u, v-\mathcal{T} v\rangle, \quad \text { for all } u, v \in C .
$$

Remark 2.8 If $\mathcal{T}$ is a nonexpansive mapping and $\langle u-\mathcal{T} u, v-\mathcal{T} v\rangle \geq 0$, for every $u, v \in C$, then $\mathcal{T}$ is a nonspreading mapping.

Lemma 2.9 Let $C$ be a nonempty closed convex subset of a real Hilbert space $H$ and let $\mathcal{T}: C \rightarrow C$ be a nonspreading mapping with $\operatorname{Fix}(\mathcal{T}) \neq \emptyset$. Then we have the following statements:

(i) $\operatorname{Fix}(\mathcal{T})=\operatorname{VI}(C, I-\mathcal{T})$;

(ii) for every $u \in C$ and $v \in \operatorname{Fix}(T)$,

$$
\left\|P_{C}(I-\lambda(I-\mathcal{T})) u-v\right\| \leq\|u-v\|, \quad \text { where } \lambda \in(0,1) .
$$


Proof To prove (i), let $x^{*} \in \operatorname{Fix}(\mathcal{T})$. Then $x^{*}=\mathcal{T} x^{*}$. Since

$$
\left\langle v-x^{*},(I-\mathcal{T}) x^{*}\right\rangle=0, \quad \forall v \in C,
$$

we have $x^{*} \in \operatorname{VI}(C, I-\mathcal{T})$, from which it follows that $\operatorname{Fix}(T) \subseteq \operatorname{VI}(C, I-\mathcal{T})$.

Next, we show $\operatorname{VI}(C, I-\mathcal{T}) \subseteq \operatorname{Fix}(\mathcal{T})$.

Let $\tilde{x} \in \operatorname{VI}(C, I-\mathcal{T})$. This implies that

$$
\langle v-\tilde{x},(I-\mathcal{T}) \tilde{x}\rangle \geq 0, \quad \forall v \in C .
$$

Let $x^{*} \in \operatorname{Fix}(\mathcal{T})$. Then, by Lemma 2.7, we obtain

$$
\left\|\mathcal{T} \tilde{x}-\mathcal{T} x^{*}\right\|^{2} \leq\left\|\tilde{x}-x^{*}\right\|^{2}+2\left|\tilde{x}-\mathcal{T} \tilde{x}, x^{*}-\mathcal{T} x^{*}\right\rangle=\left\|\tilde{x}-x^{*}\right\|^{2} .
$$

Observe that

$$
\begin{aligned}
\left\|\mathcal{T} \tilde{x}-x^{*}\right\|^{2} & =\left\|\tilde{x}-x^{*}-(I-\mathcal{T}) \tilde{x}\right\|^{2} \\
& =\left\|\tilde{x}-x^{*}\right\|^{2}-2\left(\tilde{x}-x^{*},(I-\mathcal{T}) \tilde{x}\right\rangle+\|(I-\mathcal{T}) \tilde{x}\|^{2} .
\end{aligned}
$$

From (2.1), (2.2), and (2.3), we get

$$
\|(I-\mathcal{T}) \tilde{x}\|^{2} \leq 2\left|\tilde{x}-x^{*},(I-\mathcal{T}) \tilde{x}\right\rangle \leq 0,
$$

which yields $\tilde{x} \in \operatorname{Fix}(\mathcal{T})$. Therefore $\operatorname{VI}(C, I-\mathcal{T}) \subseteq \operatorname{Fix}(\mathcal{T})$.

To prove (ii), let $u \in C$ and $v \in \operatorname{Fix}(\mathcal{T})$. Since $\mathcal{T}$ is a nonspreading mapping and we have Lemma 2.7, we get

$$
\|\mathcal{T} u-\mathcal{T} v\|^{2} \leq\|u-v\|^{2}+2\langle u-\mathcal{T} u, v-\mathcal{T} v\rangle=\|u-v\|^{2} .
$$

Thus we have

$$
\begin{aligned}
\|\mathcal{T} u-v\|^{2} & =\|u-v-(I-\mathcal{T}) u\|^{2} \\
& =\|u-v\|^{2}-2\langle u-v,(I-\mathcal{T}) u\rangle+\|(I-\mathcal{T}) u\|^{2} .
\end{aligned}
$$

From (2.4) and (2.5), we obtain

$$
\|(I-\mathcal{T}) u\|^{2} \leq 2\langle u-v,(I-\mathcal{T}) u\rangle .
$$

From (i) and Lemma 2.5, we have

$$
v \in \operatorname{Fix}(\mathcal{T})=\operatorname{VI}(C, I-\mathcal{T})=\operatorname{Fix}\left(P_{C}(I-\lambda(I-\mathcal{T}))\right) .
$$

By the nonexpansiveness of $P_{C},(2.6)$, and (2.7), we get

$$
\begin{aligned}
\left\|P_{C}(I-\lambda(I-\mathcal{T})) u-v\right\|^{2} & =\left\|P_{C}(I-\lambda(I-\mathcal{T})) u-P_{C}(I-\lambda(I-\mathcal{T})) v\right\|^{2} \\
& \leq\|(I-\lambda(I-\mathcal{T})) u-(I-\lambda(I-\mathcal{T})) v\|^{2}
\end{aligned}
$$




$$
\begin{aligned}
& =\|(u-v)-\lambda((I-\mathcal{T}) u-(I-\mathcal{T}) v)\|^{2} \\
& =\|(u-v)-\lambda(I-\mathcal{T}) u\|^{2} \\
& =\|u-v\|^{2}-2 \lambda\langle u-v,(I-\mathcal{T}) u\rangle+\lambda^{2}\|(I-\mathcal{T}) u\|^{2} \\
& \leq\|u-v\|^{2}-\lambda\|(I-\mathcal{T}) u\|^{2}+\lambda^{2}\|(I-\mathcal{T}) u\|^{2} \\
& =\|u-v\|^{2}-\lambda(1-\lambda)\|(I-\mathcal{T}) u\|^{2} \\
& \leq\|u-v\|^{2},
\end{aligned}
$$

which implies that $\left\|P_{C}(I-\lambda(I-\mathcal{T})) u-v\right\| \leq\|u-v\|$.

Lemma 2.10 ([12]) Let $C$ be a nonempty closed convex subset of a real Hilbert space H. For $i=1,2, \ldots, N$, let $\Phi_{i}: C \times C \rightarrow \mathbb{R}$ be bifunctions satisfying (A1)-(A4) with $\bigcap_{i=1}^{N} \operatorname{EP}\left(\Phi_{i}\right) \neq \emptyset$.

Then

$$
\operatorname{EP}\left(\sum_{i=1}^{N} a_{i} \Phi_{i}\right)=\bigcap_{i=1}^{N} \operatorname{EP}\left(\Phi_{i}\right),
$$

where $a_{i} \in(0,1)$ for every $i=1,2, \ldots, N$ and $\sum_{i=1}^{N} a_{i}=1$.

Lemma 2.11 ([7]) Let $C$ be a nonempty closed convex subset of $H$ and let $\Phi$ be a bifunction of $C \times C$ into $\mathbb{R}$ satisfying (A1)-(A4). Let $t>0$ and $u \in H$. Then there exists $w \in C$ such that

$$
\Phi(w, v)+\frac{1}{t}\langle v-w, w-u\rangle \geq 0, \quad \forall v \in C .
$$

Lemma 2.12 ([8]) Assume that $\Phi: C \times C \rightarrow \mathbb{R}$ satisfies (A1)-(A4). For $t>0$, define a mapping $S_{t}: H \rightarrow C$ as follows:

$$
S_{t}(x)=\left\{w \in C: \Phi(w, v)+\frac{1}{t}\langle v-w, w-u\rangle \geq 0, \forall v \in C\right\}
$$

for all $u \in H$. Then the following hold:

(i) $S_{t}$ is single-valued;

(ii) $S_{t}$ is firmly nonexpansive, i.e., for each $u, v \in H$,

$$
\left\|S_{t}(u)-S_{t}(v)\right\|^{2} \leq\left\langle S_{t}(u)-S_{t}(u), u-v\right\rangle
$$

(iii) $\operatorname{Fix}\left(S_{t}\right)=\mathrm{EP}(\Phi)$;

(iv) $\mathrm{EP}(\Phi)$ is closed and convex.

Remark 2.13 ([12]) From Lemma 2.10, it is easy to see that $\sum_{i=1}^{N} a_{i} \Phi_{i}$ satisfies (A1)-(A4). By using Lemma 2.12, we obtain

$$
\operatorname{Fix}\left(S_{t}\right)=\operatorname{EP}\left(\sum_{i=1}^{N} a_{i} \Phi_{i}\right)=\bigcap_{i=1}^{N} \operatorname{EP}\left(\Phi_{i}\right),
$$

where $a_{i} \in(0,1)$, for each $i=1,2, \ldots, N$, and $\sum_{i=1}^{N} a_{i}=1$. 


\section{Strong convergence theorem}

Theorem 3.1 Let $C$ be a nonempty closed convex subset of a real Hilbert space $H$. Let $\mathcal{F}$ be an $\alpha$-contractive mapping on $H$ and let $\mathcal{A}$ be a strongly positive linear bounded operator on $H$ with coefficient $\bar{\gamma}$ and $0<\gamma<\frac{\bar{\gamma}}{\alpha}$. Let $\mathcal{T}: C \rightarrow C$ be a nonspreading mapping. For every $i=1,2, \ldots, N$, let $\Phi_{i}: C \times C \rightarrow \mathbb{R}$ be a bifunction satisfying (A1)-(A4) with $\Omega:=\operatorname{Fix}(\mathcal{T}) \cap$ $\bigcap_{i=1}^{N} \operatorname{EP}\left(\Phi_{i}\right) \neq \emptyset$. Let $\left\{\mathcal{Z}_{n}\right\},\left\{\mathcal{Y}_{n}\right\}$, and $\left\{\mathcal{V}_{n}\right\}$ be sequences generated by $\mathcal{Z}_{1} \in H$ and

$$
\left\{\begin{array}{l}
\sum_{i=1}^{N} a_{i} \Phi_{i}\left(\mathcal{V}_{n}, y\right)+\frac{1}{\varphi_{n}}\left\langle y-\mathcal{V}_{n}, \mathcal{V}_{n}-\mathcal{Z}_{n}\right\rangle \geq 0, \quad \forall y \in C, \\
y_{n}=\theta_{n} P_{C} \mathcal{Z}_{n}+\left(1-\theta_{n}\right) \mathcal{V}_{n}, \\
\mathcal{Z}_{n+1}=\delta_{n} \gamma \mathcal{F}\left(\mathcal{Z}_{n}\right)+\left(I-\delta_{n} \mathcal{A}\right) P_{C}\left(I-\psi_{n}(I-\mathcal{T})\right) \mathcal{Y}_{n}, \quad \forall n \in \mathbb{N},
\end{array}\right.
$$

where $\left\{\delta_{n}\right\},\left\{\theta_{n}\right\},\left\{\varphi_{n}\right\},\left\{\psi_{n}\right\} \subset(0,1), 0<a_{i}<1$, for all $i=1,2, \ldots, N$. Suppose the conditions (i)-(vi) hold.

(i) $\lim _{n \rightarrow \infty} \delta_{n}=0$ and $\sum_{n=1}^{\infty} \delta_{n}=\infty$;

(ii) $0<\tau \leq \theta_{n} \leq v<1$, for some $\tau, v>0$;

(iii) $\sum_{n=1}^{\infty} \psi_{n}<\infty$;

(iv) $0<\epsilon \leq \varphi_{n} \leq \eta<1$, for some $\epsilon, \eta>0$;

(v) $\sum_{n=1}^{N} a_{i}=1$;

(vi) $\sum_{n=1}^{\infty}\left|\delta_{n+1}-\delta_{n}\right|<\infty, \sum_{n=1}^{\infty}\left|\theta_{n+1}-\theta_{n}\right|<\infty, \sum_{n=1}^{\infty}\left|\psi_{n+1}-\psi_{n}\right|<\infty$, $\sum_{n=1}^{\infty}\left|\varphi_{n+1}-\varphi_{n}\right|<\infty$

Then the sequences $\left\{\mathcal{Z}_{n}\right\},\left\{\mathcal{Y}_{n}\right\}$, and $\left\{\mathcal{V}_{n}\right\}$ converge strongly to $q=P_{\Omega}(I-\mathcal{A}+\gamma \mathcal{F}) q$.

Proof The proof of this theorem is divided into five steps.

Step 1. Claim that $\left\{\mathcal{Z}_{n}\right\}$ is a bounded sequence.

Since $\delta_{n} \rightarrow 0$ as $n \rightarrow \infty$, without loss of generality, we assume $\delta_{n}<\frac{1}{\|\cdot A\|}$, for every $n \in \mathbb{N}$. Since $\sum_{i=1}^{N} a_{i} \Phi_{i}$ satisfies (A1)-(A4) and

$$
\sum_{i=1}^{N} a_{i} \Phi_{i}\left(\mathcal{V}_{n}, y\right)+\frac{1}{\varphi_{n}}\left\langle y-\mathcal{V}_{n}, \mathcal{V}_{n}-\mathcal{Z}_{n}\right\rangle \geq 0, \quad \forall y \in C,
$$

by Lemma 2.12 and Remark 2.13, we have $\mathcal{V}_{n}=T_{\varphi_{n}} \mathcal{Z}_{n}$ and $\operatorname{Fix}\left(T_{\varphi_{n}}\right)=\bigcap_{i=1}^{N} \operatorname{EP}\left(\Phi_{i}\right)$.

From Lemma 2.5 and Lemma 2.9(i), we obtain

$$
\operatorname{Fix}(\mathcal{T})=\operatorname{Fix}\left(P_{C}\left(I-\psi_{n}(I-\mathcal{T})\right)\right)
$$

Let $z \in \Omega$. By the nonexpansiveness of $P_{C}$ and $T_{\varphi_{n}}$, we have

$$
\left\|\mathcal{Y}_{n}-z\right\| \leq \theta_{n}\left\|P_{C} \mathcal{Z}_{n}-z\right\|+\left(1-\theta_{n}\right)\left\|T_{\varphi_{n}} \mathcal{Z}_{n}-z\right\| \leq\left\|\mathcal{Z}_{n}-z\right\|
$$

From Lemma 2.6, Lemma 2.9(ii), and (3.2), we obtain

$$
\begin{aligned}
& \left\|\mathcal{Z}_{n+1}-z\right\| \\
& \quad \leq \delta_{n}\left\|\gamma \mathcal{F}\left(\mathcal{Z}_{n}\right)-\mathcal{A} z\right\|+\left\|I-\delta_{n} \mathcal{A}\right\|\left\|P_{C}\left(I-\psi_{n}(I-\mathcal{T})\right) y_{n}-z\right\| \\
& \quad \leq \delta_{n} \gamma\left\|\mathcal{F}\left(\mathcal{Z}_{n}\right)-\mathcal{F}(z)\right\|+\delta_{n}\|\gamma \mathcal{F}(z)-\mathcal{A} z\|+\left(1-\delta_{n} \bar{\gamma}\right)\left\|y_{n}-z\right\| \\
& \quad \leq \delta_{n} \gamma \alpha\left\|\mathcal{Z}_{n}-z\right\|+\delta_{n}\|\gamma \mathcal{F}(z)-\mathcal{A} z\|+\left(1-\delta_{n} \bar{\gamma}\right)\left\|\mathcal{Z}_{n}-z\right\|
\end{aligned}
$$




$$
\begin{aligned}
& =\left(1-\delta_{n}(\bar{\gamma}-\gamma \alpha)\right)\left\|\mathbb{Z}_{n}-z\right\|+\delta_{n}\|\gamma \mathcal{F}(z)-\mathcal{A} z\| \\
& \leq \max \left\{\left\|\mathcal{Z}_{1}-z\right\|, \frac{\|\gamma \mathcal{F}(z)-\mathcal{A} z\|}{\bar{\gamma}-\gamma \alpha}\right\} .
\end{aligned}
$$

By induction, we obtain $\left\|\mathcal{Z}_{n}-z\right\| \leq \max \left\{\left\|\mathcal{Z}_{1}-z\right\|, \frac{\|\gamma \mathcal{F}(z)-\mathcal{A} z\|}{\bar{\gamma}-\gamma \alpha}\right\}, \forall n \in \mathbb{N}$. It shows that $\left\{\mathcal{Z}_{n}\right\}$ is bounded and so are $\left\{\mathcal{V}_{n}\right\}$ and $\left\{\mathcal{Y}_{n}\right\}$.

Step 2. Show that $\lim _{n \rightarrow \infty}\left\|\mathcal{Z}_{n+1}-\mathcal{Z}_{n}\right\|=0$.

By the definition of $\mathcal{Z}_{n}$ and Lemma 2.6 , we obtain

$$
\begin{aligned}
& \left\|Z_{n+1}-Z_{n}\right\| \\
& \leq \delta_{n} \gamma\left\|\mathcal{F}\left(\mathcal{Z}_{n}\right)-\mathcal{F}\left(\mathcal{Z}_{n-1}\right)\right\|+\gamma\left|\delta_{n}-\delta_{n-1}\right|\left\|\mathcal{F}\left(\mathcal{Z}_{n-1}\right)\right\| \\
& +\left\|I-\delta_{n} \mathcal{A}\right\|\left\|P_{C}\left(I-\psi_{n}(I-\mathcal{T})\right) y_{n}-P_{C}\left(I-\psi_{n-1}(I-\mathcal{T})\right) y_{n-1}\right\| \\
& +\|\left(I-\delta_{n} \mathcal{A}\right) P_{C}\left(I-\psi_{n-1}(I-\mathcal{T})\right) \mathcal{y}_{n-1} \\
& -\left(I-\delta_{n-1} A\right) P_{C}\left(I-\psi_{n-1}(I-\mathcal{T})\right) y_{n-1} \| \\
& \leq \delta_{n} \gamma \alpha\left\|\mathbb{Z}_{n}-\mathcal{Z}_{n-1}\right\|+\gamma\left|\delta_{n}-\delta_{n-1}\right|\left\|\mathcal{F}\left(\mathcal{Z}_{n-1}\right)\right\| \\
& +\left(1-\delta_{n} \bar{\gamma}\right)\left\|\left(I-\psi_{n}(I-\mathcal{T})\right) \mathcal{y}_{n}-\left(I-\psi_{n-1}(I-\mathcal{T})\right) \mathcal{y}_{n-1}\right\| \\
& +\left|\delta_{n}-\delta_{n-1}\right|\left\|\mathcal{A} P_{C}\left(I-\psi_{n-1}(I-\mathcal{T})\right) \mathcal{y}_{n-1}\right\| \\
& \leq \delta_{n} \gamma \alpha\left\|\mathcal{Z}_{n}-\mathcal{Z}_{n-1}\right\|+\gamma\left|\delta_{n}-\delta_{n-1}\right|\left\|\mathcal{F}\left(\mathcal{Z}_{n-1}\right)\right\|+\left(1-\delta_{n} \bar{\gamma}\right)\left[\theta_{n}\left\|\mathcal{Z}_{n}-\mathcal{Z}_{n-1}\right\|\right. \\
& +\left|\theta_{n}-\theta_{n-1}\right|\left\|P_{C} \mathcal{Z}_{n-1}\right\|+\left(1-\theta_{n}\right)\left\|\mathcal{V}_{n}-\mathcal{V}_{n-1}\right\|+\left|\theta_{n}-\theta_{n-1}\right|\left\|\mathcal{V}_{n-1}\right\| \\
& \left.+\psi_{n}\left\|(I-\mathcal{T}) \mathcal{y}_{n}-(I-\mathcal{T}) \mathcal{y}_{n-1}\right\|+\left|\psi_{n}-\psi_{n-1}\right|\left\|(I-\mathcal{T}) \mathcal{y}_{n-1}\right\|\right] \\
& +\left|\delta_{n}-\delta_{n-1}\right|\left\|\mathcal{A} P_{C}\left(I-\psi_{n-1}(I-\mathcal{T})\right) y_{n-1}\right\| .
\end{aligned}
$$

Using the same method as in [12] (Step 2 of Theorem 3.1), we have

$$
\left\|\mathcal{V}_{n}-\mathcal{V}_{n-1}\right\| \leq\left\|\mathcal{Z}_{n}-\mathcal{Z}_{n-1}\right\|+\frac{1}{\epsilon}\left|\varphi_{n}-\varphi_{n-1}\right|\left\|\mathcal{V}_{n}-\mathcal{Z}_{n}\right\|
$$

Substitute (3.3) into (3.4) to get

$$
\begin{aligned}
& \left\|Z_{n+1}-Z_{n}\right\| \\
& \leq \delta_{n} \gamma \alpha\left\|\mathbb{Z}_{n}-\mathfrak{Z}_{n-1}\right\|+\gamma\left|\delta_{n}-\delta_{n-1}\right|\left\|\mathcal{F}\left(\mathfrak{Z}_{n-1}\right)\right\|+\left(1-\delta_{n} \bar{\gamma}\right)\left[\left\|\mathfrak{Z}_{n}-\mathfrak{Z}_{n-1}\right\|\right. \\
& +\left|\theta_{n}-\theta_{n-1}\right||| P_{C} \mathcal{Z}_{n-1}\left\|+\frac{1-\theta_{n}}{\epsilon}\left|\varphi_{n}-\varphi_{n-1}\right|\right\| \mathcal{V}_{n}-\mathcal{Z}_{n}\left\|+\left|\theta_{n}-\theta_{n-1}\right|\right\| \mathcal{V}_{n-1} \| \\
& \left.+\psi_{n}\left\|(I-\mathcal{T}) y_{n}-(I-\mathcal{T}) y_{n-1}\right\|+\left|\psi_{n}-\psi_{n-1}\right|\left\|(I-\mathcal{T}) y_{n-1}\right\|\right] \\
& +\left|\delta_{n}-\delta_{n-1}\right|\left\|\mathcal{A} P_{C}\left(I-\psi_{n-1}(I-\mathcal{T})\right) y_{n-1}\right\| \\
& \leq\left(1-\delta_{n}(\bar{\gamma}-\gamma \alpha)\right)\left\|\mathcal{Z}_{n}-\mathcal{Z}_{n-1}\right\|+(1+\gamma)\left|\delta_{n}-\delta_{n-1}\right| K+2\left|\theta_{n}-\theta_{n-1}\right| K \\
& +\frac{1}{\epsilon}\left|\varphi_{n}-\varphi_{n-1}\right| K+2 \psi_{n} K+\left|\psi_{n}-\psi_{n-1}\right| K
\end{aligned}
$$


where $K=\max _{n \in \mathbb{N}}\left\{\left\|\mathcal{V}_{n}\right\|,\left\|\mathcal{F}\left(\mathcal{Z}_{n}\right)\right\|,\left\|\mathcal{V}_{n}-\mathcal{Z}_{n}\right\|,\left\|P_{C} \mathcal{Z}_{n}\right\|,\left\|(I-\mathcal{T}) \mathcal{Y}_{n}\right\|, \| \mathcal{A} P_{C}\left(I-\psi_{n}(I-\right.\right.$ $\mathcal{T}$ )) $\left.y_{n} \|\right\}$. From (3.5), the conditions (i), (iii), (v), and Lemma 2.3, we have

$$
\lim _{n \rightarrow \infty}\left\|Z_{n+1}-Z_{n}\right\|=0
$$

Step 3. Prove that $\lim _{n \rightarrow \infty}\left\|\mathcal{V}_{n}-\mathcal{Z}_{n}\right\|=\lim _{n \rightarrow \infty}\left\|P_{C}\left(I-\psi_{n}(I-\mathcal{T})\right) \mathcal{Z}_{n}-\mathcal{Z}_{n}\right\|=0$.

To claim this, let $z \in \Omega$. Since $\mathcal{V}_{n}=T_{\varphi_{n}} Z_{n}$ and $T_{\varphi_{n}}$ is a firmly nonexpansive mapping, we have

$$
\begin{aligned}
\left\|z-T_{\varphi_{n}} \mathcal{Z}_{n}\right\|^{2} & =\left\|T_{\varphi_{n}} z-T_{\varphi_{n}} \mathcal{Z}_{n}\right\|^{2} \\
& \leq\left\langle T_{\varphi_{n}} z-T_{\varphi_{n}} Z_{n}, z-\mathcal{Z}_{n}\right\rangle \\
& =\frac{1}{2}\left(\left\|T_{\varphi_{n}} Z_{n}-z\right\|^{2}+\left\|\mathcal{Z}_{n}-z\right\|^{2}-\left\|T_{\varphi_{n}} \mathcal{Z}_{n}-\mathcal{Z}_{n}\right\|^{2}\right),
\end{aligned}
$$

from which it follows that

$$
\left\|\mathcal{V}_{n}-z\right\|^{2} \leq\left\|\mathcal{Z}_{n}-z\right\|^{2}-\left\|\mathcal{V}_{n}-\mathcal{Z}_{n}\right\|^{2}
$$

By the definition of $\mathcal{Z}_{n}$, Lemma 2.6, Lemma 2.9(ii), and (3.7), we get

$$
\begin{aligned}
&\left\|\mathcal{Z}_{n+1}-z\right\|^{2} \\
&=\left\|\delta_{n}\left(\gamma \mathcal{F}\left(\mathcal{Z}_{n}\right)-\mathcal{A} P_{C}\left(I-\psi_{n}(I-\mathcal{T})\right) y_{n}\right)+\left(P_{C}\left(I-\psi_{n}(I-\mathcal{T})\right) y_{n}-z\right)\right\|^{2} \\
& \leq\left\|P_{C}\left(I-\psi_{n}(I-\mathcal{T})\right) \mathcal{y}_{n}-z\right\|^{2} \\
&+2 \delta_{n}\left(\gamma \mathcal{F}\left(\mathcal{Z}_{n}\right)-\mathcal{A} P_{C}\left(I-\psi_{n}(I-\mathcal{T})\right) y_{n}, \mathcal{Z}_{n+1}-z\right\rangle \\
& \leq\left\|y_{n}-z\right\|^{2}+2 \delta_{n}\left\|\gamma \mathcal{F}\left(\mathcal{Z}_{n}\right)-\mathcal{A} P_{C}\left(I-\psi_{n}(I-\mathcal{T})\right) y_{n}\right\|\left\|\mathcal{Z}_{n+1}-z\right\| \\
& \leq \theta_{n}\left\|P_{C} \mathcal{Z}_{n}-z\right\|^{2}+\left(1-\theta_{n}\right)\left\|\mathcal{V}_{n}-z\right\|^{2} \\
&+2 \delta_{n}\left\|\gamma \mathcal{F}\left(\mathcal{Z}_{n}\right)-\mathcal{A} P_{C}\left(I-\psi_{n}(I-\mathcal{T})\right) y_{n}\right\|\left\|\mathcal{Z}_{n+1}-z\right\| \\
& \leq \theta_{n}\left\|\mathcal{Z}_{n}-z\right\|^{2}+\left(1-\theta_{n}\right)\left(\left\|\mathcal{Z}_{n}-z\right\|^{2}-\left\|\mathcal{V}_{n}-\mathcal{Z}_{n}\right\|^{2}\right) \\
&+2 \delta_{n}\left\|\gamma \mathcal{F}\left(\mathcal{Z}_{n}\right)-\mathcal{A} P_{C}\left(I-\psi_{n}(I-\mathcal{T})\right) y_{n}\right\|\left\|\mathcal{Z}_{n+1}-z\right\| \\
&=\left\|\mathcal{Z}_{n}-z\right\|^{2}-\left(1-\theta_{n}\right)\left\|\mathcal{V}_{n}-\mathcal{Z}_{n}\right\|^{2} \\
&+2 \delta_{n}\left\|\gamma \mathcal{F}\left(\mathcal{Z}_{n}\right)-\mathcal{A} P_{C}\left(I-\psi_{n}(I-\mathcal{T})\right) y_{n}\right\|\left\|\mathcal{Z}_{n+1}-z\right\|,
\end{aligned}
$$

which implies that

$$
\begin{aligned}
\left(1-\theta_{n}\right)\left\|\mathcal{V}_{n}-\mathcal{Z}_{n}\right\|^{2} \leq & \left(\left\|\mathcal{Z}_{n}-z\right\|+\left\|\mathcal{Z}_{n+1}-z\right\|\right)\left\|\mathcal{Z}_{n+1}-\mathcal{Z}_{n}\right\| \\
& +2 \delta_{n}\left\|\gamma \mathcal{F}\left(\mathcal{Z}_{n}\right)-\mathcal{A} P_{C}\left(I-\psi_{n}(I-\mathcal{T})\right) y_{n}\right\|\left\|\mathcal{Z}_{n+1}-z\right\| .
\end{aligned}
$$

From (3.6), the conditions (i) and (ii), this yields

$$
\lim _{n \rightarrow \infty}\left\|\mathcal{V}_{n}-\mathcal{Z}_{n}\right\|=0
$$


By Lemma 2.6 and Lemma 2.9(ii), we get

$$
\begin{aligned}
& \left\|Z_{n+1}-z\right\|^{2} \\
& \leq\left\|P_{C}\left(I-\psi_{n}(I-\mathcal{T})\right) y_{n}-z\right\|^{2} \\
& +2 \delta_{n}\left\langle\gamma \mathcal{F}\left(\mathcal{Z}_{n}\right)-\mathcal{A} P_{C}\left(I-\psi_{n}(I-\mathcal{T})\right) \mathcal{y}_{n}, \mathcal{Z}_{n+1}-z\right\rangle \\
& \leq\left\|\mathcal{Y}_{n}-z\right\|^{2}+2 \delta_{n}\left\|\gamma \mathcal{F}\left(\mathcal{Z}_{n}\right)-\mathcal{A} P_{C}\left(I-\psi_{n}(I-\mathcal{T})\right) \mathcal{y}_{n}\right\|\left\|\mathcal{Z}_{n+1}-z\right\| \\
& =\theta_{n}\left\|P_{C} \mathcal{Z}_{n}-z\right\|^{2}+\left(1-\theta_{n}\right)\left\|\mathcal{V}_{n}-z\right\|^{2}-\theta_{n}\left(1-\theta_{n}\right)\left\|P_{C} \mathcal{Z}_{n}-\mathcal{V}_{n}\right\|^{2} \\
& +2 \delta_{n}\left\|\gamma \mathcal{F}\left(\mathcal{Z}_{n}\right)-\mathcal{A} P_{C}\left(I-\psi_{n}(I-\mathcal{T})\right) \mathcal{y}_{n}\right\|\left\|\mathcal{Z}_{n+1}-z\right\| \\
& \leq\left\|\mathcal{Z}_{n}-z\right\|^{2}-\theta_{n}\left(1-\theta_{n}\right)\left\|P_{C} \mathcal{Z}_{n}-\mathcal{V}_{n}\right\|^{2} \\
& +2 \delta_{n}\left\|\gamma \mathcal{F}\left(\mathcal{Z}_{n}\right)-\mathcal{A} P_{C}\left(I-\psi_{n}(I-\mathcal{T})\right) \mathcal{y}_{n}\right\|\left\|\mathcal{Z}_{n+1}-z\right\|,
\end{aligned}
$$

from which it follows that

$$
\begin{aligned}
\theta_{n}\left(1-\theta_{n}\right)\left\|P_{C} \mathcal{Z}_{n}-\mathcal{V}_{n}\right\|^{2} \leq & \left(\left\|\mathcal{Z}_{n}-z\right\|+\left\|\mathcal{Z}_{n+1}-z\right\|\right)\left\|\mathcal{Z}_{n+1}-\mathcal{Z}_{n}\right\| \\
& +2 \delta_{n}\left\|\gamma \mathcal{F}\left(\mathcal{Z}_{n}\right)-\mathcal{A} P_{C}\left(I-\psi_{n}(I-\mathcal{T})\right) \mathcal{Y}_{n}\right\|\left\|\mathcal{Z}_{n+1}-z\right\|
\end{aligned}
$$

From (3.6), the conditions (i) and (ii), this implies that

$$
\lim _{n \rightarrow \infty}\left\|P_{C} \mathcal{Z}_{n}-\mathcal{V}_{n}\right\|=0
$$

Since

$$
\left\|P_{C} \mathcal{Z}_{n}-\mathfrak{Z}_{n}\right\| \leq\left\|P_{C} \mathcal{Z}_{n}-\mathcal{V}_{n}\right\|+\left\|\mathcal{V}_{n}-\mathfrak{Z}_{n}\right\|,
$$

using (3.8) and (3.9), we have

$$
\lim _{n \rightarrow \infty}\left\|P_{C} \mathbb{Z}_{n}-\mathfrak{Z}_{n}\right\|=0
$$

Since

$$
\left\|\mathcal{Z}_{n}-\mathfrak{Z}_{n}\right\| \leq \theta_{n}\left\|P_{C} \mathfrak{Z}_{n}-\mathfrak{Z}_{n}\right\|+\left(1-\theta_{n}\right)\left\|\mathcal{V}_{n}-\mathfrak{Z}_{n}\right\|,
$$

by (3.8) and (3.10), thus we obtain

$$
\lim _{n \rightarrow \infty}\left\|\mathcal{Y}_{n}-\mathcal{Z}_{n}\right\|=0
$$

Observe that

$$
\begin{aligned}
& \left\|\mathbb{Z}_{n}-P_{C}\left(I-\psi_{n}(I-\mathcal{T})\right) y_{n}\right\| \\
& \quad \leq\left\|\mathcal{Z}_{n}-\mathcal{Z}_{n+1}\right\|+\left\|\mathcal{Z}_{n+1}-P_{C}\left(I-\psi_{n}(I-\mathcal{T})\right) y_{n}\right\| \\
& \quad=\left\|\mathcal{Z}_{n}-\mathcal{Z}_{n+1}\right\|+\delta_{n}\left\|\gamma \mathcal{F}\left(\mathfrak{Z}_{n}\right)-\mathcal{A} P_{C}\left(I-\psi_{n}(I-\mathcal{T})\right) \mathcal{y}_{n}\right\|,
\end{aligned}
$$


which implies by (3.6) and the condition (i) that

$$
\lim _{n \rightarrow \infty}\left\|\mathfrak{Z}_{n}-P_{C}\left(I-\psi_{n}(I-\mathcal{T})\right) \mathcal{Y}_{n}\right\|=0
$$

Since

$$
\begin{aligned}
& \left\|\mathbb{Z}_{n}-P_{C}\left(I-\psi_{n}(I-\mathcal{T})\right) \mathfrak{Z}_{n}\right\| \\
& \quad \leq\left\|\mathfrak{Z}_{n}-P_{C}\left(I-\psi_{n}(I-\mathcal{T})\right) \mathcal{y}_{n}\right\|+\left\|P_{C}\left(I-\psi_{n}(I-\mathcal{T})\right) \mathcal{y}_{n}-P_{C}\left(I-\psi_{n}(I-\mathcal{T})\right) \mathfrak{Z}_{n}\right\| \\
& \quad \leq\left\|\mathfrak{Z}_{n}-P_{C}\left(I-\psi_{n}(I-\mathcal{T})\right) \mathcal{y}_{n}\right\|+\left\|\left(I-\psi_{n}(I-\mathcal{T})\right) \mathcal{y}_{n}-\left(I-\psi_{n}(I-\mathcal{T})\right) \mathfrak{Z}_{n}\right\| \\
& \quad \leq\left\|\mathfrak{Z}_{n}-P_{C}\left(I-\psi_{n}(I-\mathcal{T})\right) \mathcal{y}_{n}\right\|+\left\|\mathcal{y}_{n}-\mathfrak{Z}_{n}\right\|+\psi_{n}\left\|(I-\mathcal{T}) \mathcal{y}_{n}-(I-\mathcal{T}) \mathfrak{Z}_{n}\right\|,
\end{aligned}
$$

by (3.11), (3.12), and the condition (iii), we obtain

$$
\lim _{n \rightarrow \infty}\left\|\mathcal{Z}_{n}-P_{C}\left(I-\psi_{n}(I-\mathcal{T})\right) \mathcal{Z}_{n}\right\|=0
$$

Step 4. Show that $\limsup _{n \rightarrow \infty}\left\langle\gamma \mathcal{F}(q)-\mathcal{A} q, \mathbb{Z}_{n}-q\right\rangle \leq 0$, where $q=P_{\Omega}(I-\mathcal{A}+\gamma \mathcal{F}) q$. First, take a subsequence $\left\{\mathcal{Z}_{n_{k}}\right\}$ of $\left\{\mathcal{Z}_{n}\right\}$ such that

$$
\limsup _{n \rightarrow \infty}\left\langle\gamma \mathcal{F}(q)-\mathcal{A} q, \mathbb{Z}_{n}-q\right\rangle=\lim _{k \rightarrow \infty}\left\langle\gamma \mathcal{F}(q)-\mathcal{A} q, \mathbb{Z}_{n_{k}}-q\right\rangle
$$

Since $\left\{\mathcal{Z}_{n}\right\}$ is bounded, we can assume that $\mathcal{Z}_{n_{k}} \rightarrow \omega$ as $k \rightarrow \infty$. By (3.8), it follows that $u_{n_{k}} \rightarrow \omega$ as $k \rightarrow \infty$.

Assume $\omega \notin \operatorname{Fix}(\mathcal{T})$. Since $\operatorname{Fix}(\mathcal{T})=\operatorname{Fix}\left(P_{C}\left(I-\psi_{n_{k}}(I-\mathcal{T})\right)\right)$, we have $\omega \neq P_{C}\left(I-\psi_{n_{k}}(I-\right.$ $\mathcal{T})) \omega$. By the nonexpansiveness of $P_{C}$, the condition (iii), (3.13), and Opial's condition, we get

$$
\begin{aligned}
\liminf _{k \rightarrow \infty}\left\|\mathcal{Z}_{n_{k}}-\omega\right\|< & \liminf _{k \rightarrow \infty}\left\|\mathcal{Z}_{n_{k}}-P_{C}\left(I-\psi_{n_{k}}(I-\mathcal{T})\right) \omega\right\| \\
\leq & \liminf _{k \rightarrow \infty}\left(\left\|\mathcal{Z}_{n_{k}}-P_{C}\left(I-\psi_{n_{k}}(I-\mathcal{T})\right) \mathcal{Z}_{n_{k}}\right\|\right. \\
& \left.+\left\|P_{C}\left(I-\psi_{n_{k}}(I-\mathcal{T})\right) \mathcal{Z}_{n_{k}}-P_{C}\left(I-\psi_{n_{k}}(I-\mathcal{T})\right) \omega\right\|\right) \\
\leq & \liminf _{k \rightarrow \infty}\left(\left\|\mathcal{Z}_{n_{k}}-P_{C}\left(I-\psi_{n_{k}}(I-\mathcal{T})\right) \mathcal{Z}_{n_{k}}\right\|\right. \\
& \left.+\left\|\left(I-\psi_{n_{k}}(I-\mathcal{T})\right) \mathcal{Z}_{n_{k}}-\left(I-\psi_{n_{k}}(I-\mathcal{T})\right) \omega\right\|\right) \\
\leq & \liminf _{k \rightarrow \infty}\left(\left\|\mathcal{Z}_{n_{k}}-P_{C}\left(I-\psi_{n_{k}}(I-\mathcal{T})\right) \mathcal{Z}_{n_{k}}\right\|\right. \\
& \left.+\left\|\mathfrak{Z}_{n_{k}}-\omega\right\|+\psi_{n_{k}}\left\|(I-\mathcal{T}) \mathcal{Z}_{n_{k}}-(I-\mathcal{T}) \omega\right\|\right) \\
\leq & \liminf _{k \rightarrow \infty}\left\|\mathbb{Z}_{n_{k}}-\omega\right\| .
\end{aligned}
$$

This is a contradiction. Then we have

$$
\omega \in \operatorname{Fix}(\mathcal{T}) .
$$

By continuing the same argument as in [12] (Step 4 of Theorem 3.1), we obtain

$$
\omega \in \bigcap_{i=1}^{N} \operatorname{EP}\left(\Phi_{i}\right) .
$$


From (3.14) and (3.15), we get $\omega \in \Omega$. Since $\mathcal{Z}_{n_{k}} \rightarrow \omega$ as $k \rightarrow \infty$, by Lemma 2.1 we can conclude that

$$
\begin{aligned}
\limsup _{n \rightarrow \infty}\left\langle\gamma \mathcal{F}(q)-\mathcal{A} q, \mathbb{Z}_{n}-q\right\rangle & =\lim _{k \rightarrow \infty}\left\langle\gamma \mathcal{F}(q)-\mathcal{A} q, \mathbb{Z}_{n_{k}}-q\right\rangle \\
& =\langle\gamma \mathcal{F}(q)-\mathcal{A} q, \omega-q\rangle \\
& \leq 0 .
\end{aligned}
$$

Step 5. Finally, claim that the sequence $\left\{\mathcal{Z}_{n}\right\}$ converges strongly to $q=P_{\Omega}(I-\mathcal{A}+\gamma \mathcal{F}) q$. By Lemma 2.4, Lemma 2.6, and Lemma 2.9(ii), we obtain

$$
\begin{aligned}
&\left\|\mathcal{Z}_{n+1}-q\right\|^{2} \\
&=\left\|\delta_{n}\left(\gamma \mathcal{F}\left(\mathcal{Z}_{n}\right)-\mathcal{A} q\right)+\left(I-\delta_{n} \mathcal{A}\right)\left(P_{C}\left(I-\psi_{n}(I-\mathcal{T})\right) y_{n}-q\right)\right\|^{2} \\
& \leq\left\|\left(I-\delta_{n} \mathcal{A}\right)\left(P_{C}\left(I-\psi_{n}(I-\mathcal{T})\right) \mathcal{Y}_{n}-q\right)\right\|^{2} \\
&+2 \delta_{n}\left\langle\gamma \mathcal{F}\left(\mathcal{Z}_{n}\right)-\mathcal{A} q, \mathcal{Z}_{n+1}-q\right\rangle \\
& \leq\left(1-\delta_{n} \bar{\gamma}\right)^{2}\left\|\mathcal{Z}_{n}-q\right\|^{2}+2 \delta_{n} \gamma\left\|\mathcal{F}\left(\mathcal{Z}_{n}\right)-\mathcal{F}(q)\right\|\left\|\mathcal{Z}_{n+1}-q\right\| \\
&+2 \delta_{n}\left\langle\gamma \mathcal{F}(q)-\mathcal{A} q, \mathcal{Z}_{n+1}-q\right\rangle \\
& \leq\left(1-\delta_{n} \bar{\gamma}\right)^{2}\left(\theta_{n}\left\|P_{C} \mathcal{Z}_{n}-q\right\|^{2}+\left(1-\theta_{n}\right)\left\|\mathcal{V}_{n}-q\right\|^{2}\right) \\
&+2 \delta_{n} \gamma \alpha\left\|\mathcal{Z}_{n}-q\right\|\left\|\mathcal{Z}_{n+1}-q\right\|+2 \delta_{n}\left\langle\gamma \mathcal{F}(q)-\mathcal{A} q, \mathcal{Z}_{n+1}-q\right\rangle \\
& \leq\left(1-\delta_{n} \bar{\gamma}\right)^{2}\left\|\mathcal{Z}_{n}-q\right\|^{2}+\delta_{n} \gamma \alpha\left(\left\|\mathcal{Z}_{n}-q\right\|^{2}+\left\|\mathcal{Z}_{n+1}-q\right\|^{2}\right) \\
&+2 \delta_{n}\left\langle\gamma \mathcal{F}(q)-\mathcal{A} q, \mathcal{Z}_{n+1}-q\right\rangle,
\end{aligned}
$$

which implies that

$$
\begin{aligned}
&\left\|\mathcal{Z}_{n+1}-q\right\|^{2} \\
& \leq \frac{\left(1-\delta_{n} \bar{\gamma}\right)^{2}+\delta_{n} \gamma \alpha}{1-\delta_{n} \gamma \alpha}\left\|\mathcal{Z}_{n}-q\right\|^{2}+\frac{2 \delta_{n}}{1-\delta_{n} \gamma \alpha}\left\langle\gamma \mathcal{F}(q)-\mathcal{A} q, \mathcal{Z}_{n+1}-q\right\rangle \\
&=\left(1-\frac{2 \delta_{n}(\bar{\gamma}-\gamma \alpha)}{1-\delta_{n} \gamma \alpha}\right)\left\|\mathcal{Z}_{n}-q\right\|^{2}+\frac{2 \delta_{n}(\bar{\gamma}-\gamma \alpha)}{1-\delta_{n} \gamma \alpha}\left(\frac{\delta_{n} \bar{\gamma}^{2}}{2(\bar{\gamma}-\gamma \alpha)}\left\|\mathcal{Z}_{n}-q\right\|^{2}\right. \\
&\left.+\frac{1}{\bar{\gamma}-\gamma \alpha}\left\langle\gamma \mathcal{F}(q)-\mathcal{A} q, \mathcal{Z}_{n+1}-q\right\rangle\right) .
\end{aligned}
$$

From (3.16), the condition (i), and Lemma 2.3, we can conclude that $\left\{\mathcal{Z}_{n}\right\}$ converges strongly to $q=P_{\Omega}(I-\mathcal{A}+\gamma \mathcal{F}) q$. By (3.8) and (3.11), we see that $\left\{\mathcal{V}_{n}\right\}$ and $\left\{\mathcal{y}_{n}\right\}$ converge strongly to $q=P_{\Omega}(I-\mathcal{A}+\gamma \mathcal{F}) q$. This completes the proof.

The following corollaries are direct results from Theorem 3.1.

Corollary 3.2 Let C be a nonempty closed convex subset of a real Hilbert space H. Let $\mathcal{F}$ be an $\alpha$-contractive mapping on $H$ and let $A$ be a strongly positive linear bounded operator on $H$ with coefficient $\bar{\gamma}$ and $0<\gamma<\frac{\bar{\gamma}}{\alpha}$. Let $\mathcal{T}: C \rightarrow C$ be a nonspreading mapping. Let 
$\Phi: C \times C \rightarrow \mathbb{R}$ be a bifunction satisfying (A1)-(A4) with $\Omega:=\operatorname{Fix}(\mathcal{T}) \cap \operatorname{EP}(\Phi) \neq \emptyset$. Let

$\left\{\mathcal{Z}_{n}\right\},\left\{\mathcal{Y}_{n}\right\}$, and $\left\{\mathcal{V}_{n}\right\}$ be sequences generated by $\mathcal{Z}_{1} \in H$ and

$$
\left\{\begin{array}{l}
\Phi\left(\mathcal{V}_{n}, y\right)+\frac{1}{\varphi_{n}}\left\langle y-\mathcal{V}_{n}, \mathcal{V}_{n}-\mathcal{Z}_{n}\right\rangle \geq 0, \quad \forall y \in C, \\
\mathcal{Y}_{n}=\theta_{n} P_{C} \mathcal{Z}_{n}+\left(1-\theta_{n}\right) \mathcal{V}_{n}, \\
\mathcal{Z}_{n+1}=\delta_{n} \gamma \mathcal{F}\left(\mathcal{Z}_{n}\right)+\left(I-\delta_{n} \mathcal{A}\right) P_{C}\left(I-\psi_{n}(I-\mathcal{T})\right) y_{n}, \quad \forall n \in \mathbb{N},
\end{array}\right.
$$

where $\left\{\delta_{n}\right\},\left\{\theta_{n}\right\},\left\{\varphi_{n}\right\},\left\{\psi_{n}\right\} \subseteq(0,1)$. Suppose the conditions (i)-(vi) hold.

(i) $\lim _{n \rightarrow \infty} \delta_{n}=0$ and $\sum_{n=1}^{\infty} \delta_{n}=\infty$;

(ii) $0<\tau \leq \theta_{n} \leq v<1$, for some $\tau, v>0$;

(iii) $\sum_{n=1}^{\infty} \psi_{n}<\infty$;

(iv) $0<\epsilon \leq \varphi_{n} \leq \eta<1$, for some $\epsilon, \eta>0$;

(v) $\sum_{n=1}^{\infty}\left|\delta_{n+1}-\delta_{n}\right|<\infty, \sum_{n=1}^{\infty}\left|\theta_{n+1}-\theta_{n}\right|<\infty, \sum_{n=1}^{\infty}\left|\psi_{n+1}-\psi_{n}\right|<\infty$, $\sum_{n=1}^{\infty}\left|\varphi_{n+1}-\varphi_{n}\right|<\infty$.

Then the sequences $\left\{\mathcal{Z}_{n}\right\},\left\{\mathcal{Y}_{n}\right\}$, and $\left\{\mathcal{V}_{n}\right\}$ converge strongly to $q=P_{\Omega}(I-\mathcal{A}+\gamma \mathcal{F}) q$.

Proof Put $\Phi=\Phi_{i}$, for all $i=1,2, \ldots, N$. Using Theorem 3.1, the desired result is obtained.

In 2007, Plubtieng and Punpaeng [19] introduced the general iterative method for an equilibrium problem and a nonexpansive mapping in Hilbert spaces. Let $S$ be a nonexpansive mapping on $H$ with $\operatorname{Fix}(S) \cap \operatorname{EP}(F) \neq \emptyset$. With an initial value $z_{1} \in H$, the sequences $\left\{z_{n}\right\}$ and $\left\{v_{n}\right\}$ are generated by

$$
\left\{\begin{array}{l}
F\left(v_{n}, y\right)+\frac{1}{\varphi_{n}}\left\langle y-v_{n}, v_{n}-z_{n}\right\rangle \geq 0, \quad \forall y \in H \\
z_{n+1}=\alpha_{n} \gamma f\left(z_{n}\right)+\left(I-\alpha_{n} A\right) S v_{n}, \quad \forall n \in \mathbb{N}
\end{array}\right.
$$

where $\left\{r_{n}\right\} \subset(0, \infty)$ and $\alpha_{n} \subset[0,1]$ satisfy some appropriate conditions. Then $\left\{z_{n}\right\}$ and $\left\{v_{n}\right\}$ converge strongly to a point $z$, where $z=P_{\operatorname{Fix}(S) \cap \operatorname{EP}(F)}(I-A+\gamma f)(z)$.

Later, in 2010, Ceng et al. [11] studied the iterative scheme for equilibrium problem and an infinite family of nonexpansive mappings. Let $0<\gamma \alpha<\tilde{\gamma}$. Let $\left\{\alpha_{n}\right\}$ and $\left\{\gamma_{n}\right\}$ be sequences in $(0,1)$. Starting with $z_{1} \in H$, the sequences $\left\{z_{n}\right\}$ and $\left\{u_{n}\right\}$ are generated by the following iterative scheme:

$$
\left\{\begin{array}{l}
F\left(u_{n}, y\right)+\frac{1}{r_{n}}\left\langle y-u_{n}, u_{n}-z_{n}\right\rangle \geq 0, \quad \forall y \in H, \\
v_{n}=\left(1-\gamma_{n}\right) z_{n}+\gamma_{n} W_{n} u_{n}, \\
z_{n+1}=\alpha_{n} \gamma f\left(v_{n}\right)+\left(I-\alpha_{n} A\right) W_{n} v_{n},
\end{array}\right.
$$

where $W_{n}$ is a $W$-mapping generated by an infinite family of nonexpansive mappings and infinite real numbers. Then, under some suitable conditions, the sequences $\left\{z_{n}\right\}$ and $\left\{u_{n}\right\}$ converge strongly to $z^{*}=P_{\bigcap_{n=1}^{\infty} F\left(T_{n}\right) \cap \operatorname{EP}(\phi)} \tilde{f}\left(z^{*}\right)$, where $\tilde{f}=I-\theta(A-\gamma f)$.

Remark 3.3 For Corollary 3.2, we prove the strong convergence theorem for equilibrium problem and a nonspreading mapping. Motivated by the results of Ceng et al. [11] and Plubtieng and Punpaeng [19], we consider the following statements, different from this work. 
(i) We investigate the iterative algorithm for a nonspreading mapping instead of using a nonexpansive mapping.

(ii) We study the general iterative method by using the sequence $y_{n}=\theta_{n} P_{C} \mathcal{Z}_{n}+\left(1-\theta_{n}\right) \mathcal{V}_{n}$

Corollary 3.4 Let $C$ be a nonempty closed convex subset of a real Hilbert space H. Let $\mathcal{F}$ be an $\alpha$-contractive mapping on $H$ and let $\mathcal{A}: H \rightarrow H$ be a strongly positive linear bounded operator with coefficient $\bar{\gamma}$ and $0<\gamma<\frac{\bar{\gamma}}{\alpha}$. Let $\mathcal{T}: C \rightarrow C$ be a nonspreading mapping with $\operatorname{Fix}(\mathcal{T}) \neq \emptyset$. Let $\left\{\mathcal{Z}_{n}\right\}$ be the sequence generated by $\mathcal{Z}_{1} \in H$ and

$$
\mathcal{Z}_{n+1}=\delta_{n} \gamma \mathcal{F}\left(\mathcal{Z}_{n}\right)+\left(I-\delta_{n} \mathcal{A}\right) P_{C}\left(I-\psi_{n}(I-\mathcal{T})\right) P_{C} \mathcal{Z}_{n}, \quad \forall n \in \mathbb{N},
$$

where $\left\{\delta_{n}\right\},\left\{\psi_{n}\right\} \subseteq(0,1)$. Suppose the conditions (i)-(vi) hold.

(i) $\lim _{n \rightarrow \infty} \delta_{n}=0$ and $\sum_{n=1}^{\infty} \delta_{n}=\infty$;

(ii) $\sum_{n=1}^{\infty} \psi_{n}<\infty$;

(iii) $\sum_{n=1}^{\infty}\left|\delta_{n+1}-\delta_{n}\right|<\infty, \sum_{n=1}^{\infty}\left|\psi_{n+1}-\psi_{n}\right|<\infty$.

Then the sequence $\left\{\mathcal{Z}_{n}\right\}$ converges strongly to $q=P_{\mathrm{Fix}(\mathcal{T})}(I-\mathcal{A}+\gamma \mathcal{F}) q$.

Proof Take $\Phi_{i}=0$, for every $i=1,2, \ldots, N$. Then we have $\mathcal{V}_{n}=P_{C} \mathcal{Z}_{n}$, for every $n \in \mathbb{N}$. The result of Corollary 3.4 can be obtained by Theorem 3.1.

\section{Applications}

By means of our main result, we obtain the strong convergence theorem for a finite family of nonspreading mappings and a finite family of equilibrium problems in the setting of Hilbert space. To prove this, the following definitions, remarks, and lemmas are needed.

Definition 4.1 A mapping $\mathcal{T}$ is quasi-nonexpansive if

$$
\|\mathcal{T} x-p\| \leq\|x-p\|, \quad \text { for every } x \in C \text { and } p \in \operatorname{Fix}(\mathcal{T}) .
$$

Remark 4.1 If $\mathcal{T}: C \rightarrow C$ is nonspreading with $\operatorname{Fix}(\mathcal{T}) \neq \emptyset$, then $\mathcal{T}$ is quasi-nonexpansive.

Example 4.2 Let an inner product $\langle\cdot, \cdot\rangle: \mathbb{R}^{2} \times \mathbb{R}^{2} \rightarrow \mathbb{R}$ be defined by $\langle u, v\rangle=u \cdot v=u_{1} v_{1}+$ $u_{2} v_{2}$ and a usual norm $\|\cdot\|: \mathbb{R}^{2} \rightarrow \mathbb{R}$ be given by $\|u\|=\sqrt{u_{1}^{2}+u_{2}^{2}}$, for all $u=\left(u_{1}, u_{2}\right), v=$ $\left(v_{1}, v_{2}\right) \in \mathbb{R}^{2}$. Let $I=[1,100]$ and let $\mathcal{T}: I^{2} \rightarrow I^{2}$ be defined by

$$
\mathcal{T} u=\left(\frac{u_{1}+1}{2}, \frac{5 u_{2}+1}{6}\right), \quad \text { for all } u=\left(u_{1}, u_{2}\right) \in I^{2} .
$$

First, we show that $\mathcal{T}$ is a nonspreading mapping.

For every $u, v \in I^{2}$, we obtain

$$
\begin{aligned}
\|\mathcal{T} u-\mathcal{T} v\|^{2} & =\left\|\left(\frac{u_{1}+1}{2}, \frac{5 u_{2}+1}{6}\right)-\left(\frac{v_{1}+1}{2}, \frac{5 v_{2}+1}{6}\right)\right\|^{2} \\
& =\left\|\left(\frac{1}{2}\left(u_{1}-v_{1}\right), \frac{5}{6}\left(u_{2}-v_{2}\right)\right)\right\|^{2} \\
& =\frac{1}{4}\left(u_{1}-v_{1}\right)^{2}+\frac{25}{36}\left(u_{2}-v_{2}\right)^{2}
\end{aligned}
$$


and

$$
\begin{aligned}
2\langle u & -\mathcal{T} u, v-\mathcal{T} v\rangle \\
& =2\left\langle\left(u_{1}, u_{2}\right)-\left(\frac{u_{1}+1}{2}, \frac{5 u_{2}+1}{6}\right),\left(v_{1}, v_{2}\right)-\left(\frac{v_{1}+1}{2}, \frac{5 v_{2}+1}{6}\right)\right\rangle \\
& =2\left\langle\left(\frac{u_{1}-1}{2}, \frac{u_{2}-1}{6}\right),\left(\frac{v_{1}-1}{2}, \frac{v_{2}-1}{6}\right)\right\rangle \\
& =2\left(\frac{u_{1}-1}{2}, \frac{u_{2}-1}{6}\right) \cdot\left(\frac{v_{1}-1}{2}, \frac{v_{2}-1}{6}\right) \\
& =2\left[\left(\frac{u_{1}-1}{2}\right)\left(\frac{v_{1}-1}{2}\right)+\left(\frac{u_{2}-1}{6}\right)\left(\frac{v_{2}-1}{6}\right)\right] \\
& =\frac{\left(u_{1}-1\right)\left(v_{1}-1\right)}{2}+\frac{\left(u_{2}-1\right)\left(v_{2}-1\right)}{18} \\
& \geq 0 .
\end{aligned}
$$

This yields

$$
\begin{aligned}
\|u-v\|^{2}+2\langle u-\mathcal{T} u, v-\mathcal{T} v\rangle & \geq\|u-v\|^{2} \\
& =\left\|\left(u_{1}-v_{1}, u_{2}-v_{2}\right)\right\|^{2} \\
& =\left(u_{1}-v_{1}\right)^{2}+\left(u_{2}-v_{2}\right)^{2} \\
& >\frac{1}{4}\left(u_{1}-v_{1}\right)^{2}+\frac{25}{36}\left(u_{2}-v_{2}\right)^{2} \\
& =\|\mathcal{T} u-T v\|^{2} .
\end{aligned}
$$

Then $\mathcal{T}$ is a nonspreading mapping and we observe that $\operatorname{Fix}(\mathcal{T})=\{\mathbf{1}\}$, where $\mathbf{1}=(1,1)$. For every $u \in I \times I$ and $\mathbf{1} \in \operatorname{Fix}(\mathcal{T})$, from (4.1), we have

$$
\begin{aligned}
\|\mathcal{T} u-\mathcal{T} \mathbf{1}\|^{2} & \leq\|u-\mathbf{1}\|^{2}+2\langle u-\mathcal{T} u, \mathbf{1}-\mathcal{T} \mathbf{1}\rangle \\
& =\|u-\mathbf{1}\|^{2} .
\end{aligned}
$$

Therefore $\mathcal{T}$ is a quasi-nonexpansive mapping.

The following example shows that the converse of Remark 4.1 does not hold.

Example 4.3 Let $I=[0,2]$ and let $\mathcal{T}: I^{2} \rightarrow I^{2}$ be defined by

$$
\mathcal{T} u= \begin{cases}\left(\frac{u_{1}+2}{2}, \frac{u_{2}+2}{2}\right) & \text { if } u \in(1,2] \times(1,2] \\ \left(\frac{u_{1}}{2}, \frac{u_{2}}{2}\right) & \text { if } u \in[0,1] \times[0,1]\end{cases}
$$

First, show that $\mathcal{T}$ is quasi-nonexpansive for all $u \in I^{2}$.

Observe that $\operatorname{Fix}(\mathcal{T})=\{2\}$ if $x \in(1,2] \times(1,2]$ and $\operatorname{Fix}(\mathcal{T})=\{0\}$ if $u \in[0,1] \times[0,1]$, where $\mathbf{2}=(2,2)$ and $\mathbf{0}=(0,0)$. 
For any $u \in(1,2] \times(1,2]$, we have

$$
\begin{aligned}
\left\|\left(\frac{u_{1}+2}{2}, \frac{u_{2}+2}{2}\right)-(2,2)\right\| & =\left\|\left(\frac{u_{1}-2}{2}, \frac{u_{2}-2}{2}\right)\right\| \\
& =\frac{1}{2}\left\|\left(u_{1}-2, u_{2}-2\right)\right\| \\
& =\frac{1}{2}\left\|\left(u_{1}, u_{2}\right)-(2,2)\right\| \\
& <\|u-2\| .
\end{aligned}
$$

For every $u \in[0,1] \times[0,1]$, we obtain

$$
\begin{aligned}
\left\|\left(\frac{u_{1}}{2}, \frac{u_{2}}{2}\right)-(0,0)\right\| & =\left\|\left(\frac{u_{1}}{2}, \frac{u_{2}}{2}\right)\right\| \\
& =\frac{1}{2}\left\|\left(u_{1}, u_{2}\right)\right\| \\
& <\|u\| .
\end{aligned}
$$

Therefore $\mathcal{T}$ is a quasi-nonexpansive for all $u \in I^{2}$.

Choose $u=\left(\frac{3}{2}, \frac{3}{2}\right)$ and $v=\left(\frac{1}{2}, \frac{1}{2}\right)$, we have

$$
\begin{aligned}
\left\|\mathcal{T}\left(\frac{3}{2}, \frac{3}{2}\right)-\mathcal{T}\left(\frac{1}{2}, \frac{1}{2}\right)\right\|^{2} & =\left\|\left(\frac{7}{4}, \frac{7}{4}\right)-\left(\frac{1}{4}, \frac{1}{4}\right)\right\|^{2} \\
& =\left\|\left(\frac{6}{4}, \frac{6}{4}\right)\right\|^{2}=\left(\frac{6}{4}\right)^{2}+\left(\frac{6}{4}\right)^{2}=\frac{9}{2}
\end{aligned}
$$

Thus we get

$$
\begin{aligned}
\| u & -v \|^{2}+2\langle u-\mathcal{T} u, v-\mathcal{T} v\rangle \\
& =\left\|\left(\frac{3}{2}, \frac{3}{2}\right)-\left(\frac{1}{2}, \frac{1}{2}\right)\right\|^{2}+2\left\langle\left(\frac{3}{2}, \frac{3}{2}\right)-\mathcal{T}\left(\left(\frac{3}{2}, \frac{3}{2}\right)\right),\left(\frac{1}{2}, \frac{1}{2}\right)-\mathcal{T}\left(\frac{1}{2}, \frac{1}{2}\right)\right\rangle \\
& =\|(1,1)\|^{2}+2\left\langle\left(\frac{3}{2}, \frac{3}{2}\right)-\left(\frac{7}{4}, \frac{7}{4}\right),\left(\frac{1}{2}, \frac{1}{2}\right)-\left(\frac{1}{4}, \frac{1}{4}\right)\right\rangle \\
& =2+2\left(\frac{3}{2}-\frac{7}{4}, \frac{3}{2}-\frac{7}{4}\right) \cdot\left(\frac{1}{2}-\frac{1}{4}, \frac{1}{2}-\frac{1}{4}\right) \\
& =2+2\left(-\frac{1}{4},-\frac{1}{4}\right) \cdot\left(\frac{1}{4}, \frac{1}{4}\right) \\
& =2-\frac{2}{8}=\frac{14}{8} .
\end{aligned}
$$

Hence we have

$$
\|\mathcal{T} u-\mathcal{T} v\|^{2}>\|u-v\|^{2}+2\langle u-\mathcal{T} u, v-\mathcal{T} v\rangle
$$

By changing $\mathcal{T}$ from a nonspreading mapping to a quasi-nonexpansive mapping with $\operatorname{Fix}(\mathcal{T}) \neq \emptyset$, we obtain the same result as shown in Lemma 2.9. 
Remark 4.4 Let $C$ be a nonempty closed convex subset of a real Hilbert space $H$ and let $\mathcal{T}: C \rightarrow C$ be a quasi-nonexpansive mapping with $\operatorname{Fix}(\mathcal{T}) \neq \emptyset$. Then we have the following statement:

(i) $\operatorname{Fix}(\mathcal{T})=\operatorname{VI}(C, I-\mathcal{T})$;

(ii) for every $u \in C$ and $v \in \operatorname{Fix}(\mathcal{T})$,

$$
\left\|P_{C}(I-\lambda(I-\mathcal{T})) u-v\right\| \leq\|u-v\|, \quad \text { where } \lambda \in(0,1) .
$$

Definition $4.2([24])$ Let $C$ be a nonempty convex subset of a real Banach space. Let $\left\{T_{i}\right\}_{i=1}^{N}$ be a finite family of (nonexpansive) mappings of $C$ into itself. For each $j=1,2, \ldots$, let $\alpha_{j}=\left(\alpha_{1}^{j}, \alpha_{2}^{j}, \alpha_{3}^{j}\right) \in I \times I \times I$ where $I=[0,1]$ and $\alpha_{1}^{j}+\alpha_{2}^{j}+\alpha_{3}^{j}=1$. Define the mapping $S: C \rightarrow C$ as follows:

$$
\begin{aligned}
& U_{0}=I, \\
& U_{1}=\alpha_{1}^{1} T_{1} U_{0}+\alpha_{2}^{1} U_{0}+\alpha_{3}^{1} I, \\
& U_{2}=\alpha_{1}^{2} T_{2} U_{1}+\alpha_{2}^{2} U_{1}+\alpha_{3}^{2} I, \\
& U_{3}=\alpha_{1}^{3} T_{3} U_{2}+\alpha_{2}^{3} U_{2}+\alpha_{3}^{3} I, \\
& \vdots \\
& U_{N-1}=\alpha_{1}^{N-1} T_{N-1} U_{N-2}+\alpha_{2}^{N-1} U_{N-2}+\alpha_{3}^{N-1} I, \\
& S=U_{N}=\alpha_{1}^{N} T_{N} U_{N-1}+\alpha_{2}^{N} U_{N-1}+\alpha_{3}^{N} I .
\end{aligned}
$$

This mapping is called the $S$-mapping generated by $T_{1}, T_{2}, \ldots, T_{N}$ and $\alpha_{1}, \alpha_{2}, \ldots, \alpha_{N}$.

Lemma 4.5 ([25]) Let $C$ be a nonempty closed convex subset of a real Hilbert space $H$. Let $\left\{T_{i}\right\}_{i=1}^{N}$ be a finite family of nonspreading mappings of C into itself with $\bigcap_{i=1}^{N} \operatorname{Fix}\left(T_{i}\right) \neq \emptyset$ and let $\alpha_{j}=\left(\alpha_{1}^{j}, \alpha_{2}^{j}, \alpha_{3}^{j}\right) \in I \times I \times I$ where $I=[0,1], \alpha_{1}^{j}+\alpha_{2}^{j}+\alpha_{3}^{j}=1, \alpha_{1}^{j}, \alpha_{3}^{j} \in(0,1)$ for all $j=1,2, \ldots, N-1$ and $\alpha_{1}^{N} \in(0,1], \alpha_{3}^{N} \in[0,1), \alpha_{2}^{j} \in(0,1)$ for all $j=1,2, \ldots, N$. Let $S$ be the $S$-mapping generated by $T_{1}, T_{2}, \ldots, T_{N}$ and $\alpha_{1}, \alpha_{2}, \ldots, \alpha_{N}$. Then $\operatorname{Fix}(S)=\bigcap_{i=1}^{N} \operatorname{Fix}\left(T_{i}\right)$ and $S$ is a quasi-nonexpansive mapping.

Theorem 4.6 Let $C$ be a nonempty closed convex subset of a real Hilbert space H. Let $\mathcal{F}: C \rightarrow C$ be an $\alpha$-contractive mapping, let $\mathcal{A}: C \rightarrow C$ be a strongly positive linear bounded operator with coefficient $\bar{\gamma}$ and $0<\gamma<\frac{\bar{\gamma}}{\alpha}$. For $i=1,2, \ldots, \bar{N}$, let $\Phi_{i}: C \times C \rightarrow \mathbb{R}$ be a bifunction satisfying (A1)-(A4). Let $\mathcal{T}_{i}: C \rightarrow C$, for $i=1,2, \ldots, N$ be a finite family of nonspreading mappings with $\Omega:=\bigcap_{i=1}^{N} \operatorname{Fix}\left(\mathcal{T}_{i}\right) \cap \bigcap_{i=1}^{\bar{N}} \operatorname{EP}\left(\Phi_{i}\right) \neq \emptyset$. Let $\rho_{j}=\left(\alpha_{1}^{j}, \alpha_{2}^{j}, \alpha_{3}^{j}\right) \in I \times I \times I$, $j=1,2, \ldots, N$, where $I=[0,1], \alpha_{1}^{j}+\alpha_{2}^{j}+\alpha_{3}^{j}=1, \alpha_{1}^{j}, \alpha_{3}^{j} \in(0,1)$ for all $j=1,2, \ldots, N-1$ and $\alpha_{1}^{N} \in(0,1], \alpha_{3}^{N} \in[0,1), \alpha_{2}^{j} \in(0,1)$ for all $j=1,2, \ldots, N$, and let $S$ be the $S$-mapping generated by $\mathcal{T}_{1}, \mathcal{T}_{2}, \ldots, \mathcal{T}_{N}$ and $\rho_{1}, \rho_{2}, \ldots, \rho_{N}$. Let $\left\{\mathcal{Z}_{n}\right\},\left\{\mathcal{Y}_{n}\right\}$, and $\left\{\mathcal{V}_{n}\right\}$ be sequences generated by $Z_{1} \in H$ and

$$
\left\{\begin{array}{l}
\sum_{i=1}^{\bar{N}} a_{i} \Phi_{i}\left(\mathcal{V}_{n}, y\right)+\frac{1}{\varphi_{n}}\left\langle y-\mathcal{V}_{n}, \mathcal{V}_{n}-\mathcal{Z}_{n}\right\rangle \geq 0, \quad \forall y \in C, \\
y_{n}=\theta_{n} P_{C} \mathcal{Z}_{n}+\left(1-\theta_{n}\right) \mathcal{V}_{n}, \\
\mathcal{Z}_{n+1}=\delta_{n} \gamma \mathcal{F}\left(\mathcal{Z}_{n}\right)+\left(I-\delta_{n} \mathcal{A}\right) P_{C}\left(I-\psi_{n}(I-S)\right) \mathcal{Y}_{n}, \quad \forall n \in \mathbb{N},
\end{array}\right.
$$


where $\left\{\delta_{n}\right\},\left\{\theta_{n}\right\},\left\{\varphi_{n}\right\},\left\{\psi_{n}\right\} \subseteq(0,1), 0<a_{i}<1$, for all $i=1,2, \ldots, \bar{N}$. Suppose the conditions (i)-(vi) hold.

(i) $\lim _{n \rightarrow \infty} \delta_{n}=0$ and $\sum_{n=1}^{\infty} \delta_{n}=\infty$;

(ii) $0<\tau \leq \theta_{n} \leq v<1$, for some $\tau, v>0$;

(iii) $\sum_{n=1}^{\infty} \psi_{n}<\infty$;

(iv) $0<\epsilon \leq \varphi_{n} \leq \eta<1$, for some $\epsilon, \eta>0$;

(v) $\sum_{n=1}^{\bar{N}} a_{i}=1$;

(vi) $\sum_{n=1}^{\infty}\left|\delta_{n+1}-\delta_{n}\right|<\infty, \sum_{n=1}^{\infty}\left|\theta_{n+1}-\theta_{n}\right|<\infty, \sum_{n=1}^{\infty}\left|\psi_{n+1}-\psi_{n}\right|<\infty$, $\sum_{n=1}^{\infty}\left|\varphi_{n+1}-\varphi_{n}\right|<\infty$.

Then the sequences $\left\{\mathcal{Z}_{n}\right\},\left\{\mathcal{Y}_{n}\right\}$, and $\left\{\mathcal{V}_{n}\right\}$ converge strongly to $q=P_{\Omega}(I-\mathcal{A}+\gamma \mathcal{F}) q$.

Proof Using Remark 4.4, Lemma 4.5, and the same method as in Theorem 3.1, we have the desired conclusion.

Remark 4.7 Theorem 4.6 can be considered as an improvement of Theorem 3.1 in the result of Tian and Jin [26] in the sense that some conditions are not assumed.

(i) $T_{\omega}:=(1-\omega) I+\omega T, \omega \in\left(0, \frac{1}{2}\right)$,

(ii) $T$ is demi-closed on $H$,

where $T$ is a quasi-nonexpansive mapping on $H$.

\section{Examples for equilibrium problems and numerical results}

In this section, the numerical examples are given for supporting Theorem 3.1. Using these examples, we see that our iteration for the combination of equilibrium problem converges faster than our iteration for the classical equilibrium problem.

Example 5.1 Let the mappings $\mathcal{A}: \mathbb{R} \rightarrow \mathbb{R}, \mathcal{F}: \mathbb{R} \rightarrow \mathbb{R}$, be defined by

$$
\begin{aligned}
& \mathcal{A} x=\frac{x}{2}, \\
& \mathcal{F} x=\frac{x}{4}, \quad \text { for all } x \in \mathbb{R} .
\end{aligned}
$$

For every $i=1,2, \ldots, N$, let $\Phi_{i}:[1,100] \times[1,100] \rightarrow \mathbb{R}$ and $\mathcal{T}:[1,100] \rightarrow[1,100]$ be defined by

$$
\begin{aligned}
& \mathcal{T} x=\frac{2 x+5}{7}, \\
& \Phi_{i}(x, y)=i(y-x)(y+2 x-3), \quad \text { for all } x, y \in[1,100] .
\end{aligned}
$$

Put $a_{i}=\frac{4}{5^{i}}+\frac{1}{N 5^{N}}$, for every $i=1,2, \ldots, N$. Let $\gamma=\frac{1}{3}, \delta_{n}=\frac{1}{3 n}, \theta_{n}=\frac{n}{2 n+3}, \varphi_{n}=\frac{2 n}{3 n+2}$, and $\psi_{n}=\frac{1}{n^{2}}$ for every $n \in \mathbb{N}$. Let the initial values be defined as in the following cases:

(i) $Z_{1}=50, N=1$, and $n=10$,

(ii) $Z_{1}=50$ and $n=N=10$.

Then, for both cases, the sequences $\left\{\mathcal{Z}_{n}\right\},\left\{\mathcal{Y}_{n}\right\}$, and $\left\{\mathcal{V}_{n}\right\}$ converge strongly to 1 .

Solution. It is obvious that $\mathcal{T}$ is a nonspreading mapping and $\operatorname{Fix}(\mathcal{T})=\{1\}$.

Since $a_{i}=\frac{4}{5^{i}}+\frac{1}{N 5^{N}}$, we obtain

$$
\sum_{i=1}^{N} a_{i} \Phi_{i}(x, y)=\sum_{i=1}^{N}\left(\frac{4}{5^{i}}+\frac{1}{N 5^{N}}\right) i(y-x)(y+2 x-3)=\mu(y-x)(y+2 x-3),
$$


where $\mu=\sum_{i=1}^{N}\left(\frac{4}{5^{i}}+\frac{1}{N 5^{N}}\right) i$. It is clear that $\sum_{i=1}^{N} a_{i} \Phi_{i}$ satisfies all conditions in Theorem 3.1 and $\operatorname{EP}\left(\sum_{i=1}^{N} a_{i} \Phi_{i}\right)=\bigcap_{i=1}^{N} \operatorname{EP}\left(\Phi_{i}\right)=\{1\}$. Then we have

$$
\operatorname{Fix}(\mathcal{T}) \cap \bigcap_{i=1}^{N} \operatorname{EP}\left(\Phi_{i}\right)=\{1\} .
$$

Observe that

$$
\begin{aligned}
0 \leq \sum_{i=1}^{N} a_{i} \Phi_{i}\left(\mathcal{V}_{n}, y\right)+\frac{1}{\varphi_{n}}\left\langle y-\mathcal{V}_{n}, \mathcal{V}_{n}-\mathcal{Z}_{n}\right\rangle \\
=\mu\left(y-\mathcal{V}_{n}\right)\left(y+2 \mathcal{V}_{n}-3\right)+\frac{1}{\varphi_{n}}\left(y-\mathcal{V}_{n}\right)\left(\mathcal{V}_{n}-\mathcal{Z}_{n}\right) \\
\Leftrightarrow \quad 0 \leq \mu \varphi_{n}\left(y-\mathcal{V}_{n}\right)\left(y+2 \mathcal{V}_{n}-3\right)+\left(y-\mathcal{V}_{n}\right)\left(\mathcal{V}_{n}-\mathcal{Z}_{n}\right) \\
=\mu \varphi_{n} y^{2}+\left(\mu \mathcal{V}_{n} \varphi_{n}+\mathcal{V}_{n}-\mathcal{Z}_{n}-3 \mu \varphi_{n}\right) y \\
+3 \mu \varphi_{n} \mathcal{V}_{n}-\mathcal{V}_{n}^{2}-2 \mu \varphi_{n} \mathcal{V}_{n}^{2}+\mathcal{V}_{n} \mathcal{Z}_{n} .
\end{aligned}
$$

Let $G(y)=\mu \varphi_{n} y^{2}+\left(\mu \mathcal{V}_{n} \varphi_{n}+\mathcal{V}_{n}-\mathcal{Z}_{n}-3 \mu \varphi_{n}\right) y+3 \mu \varphi_{n} \mathcal{V}_{n}-\mathcal{V}_{n}^{2}-2 \mu \varphi_{n} \mathcal{V}_{n}^{2}+\mathcal{V}_{n} \mathcal{Z}_{n} . G(y)$ is a quadratic function of $y$ with coefficients $a=\mu \varphi_{n}, b=\mu \mathcal{V}_{n} \varphi_{n}+\mathcal{V}_{n}-\mathcal{Z}_{n}-3 \mu \varphi_{n}$, and $c=3 \mu \varphi_{n} \mathcal{V}_{n}-\mathcal{V}_{n}^{2}-2 \mu \varphi_{n} \mathcal{V}_{n}^{2}+\mathcal{V}_{n} \mathcal{Z}_{n}$. Determine the discriminant $\Delta$ of $G$ as follows:

$$
\begin{aligned}
\Delta= & b^{2}-4 a c \\
= & \left(\mu \mathcal{V}_{n} \varphi_{n}+\mathcal{V}_{n}-\mathcal{Z}_{n}-3 \mu \varphi_{n}\right)^{2}-4\left(\mu \varphi_{n}\right)\left(3 \mu \varphi_{n} \mathcal{V}_{n}-\mathcal{V}_{n}^{2}-2 \mu \varphi_{n} \mathcal{V}_{n}^{2}+\mathcal{V}_{n} \mathcal{Z}_{n}\right) \\
= & 9 \mu^{2} \varphi_{n}^{2}-6 \mu \varphi_{n} \mathcal{V}_{n}-18 \mu^{2} \varphi_{n}^{2} \mathcal{V}_{n}+\mathcal{V}_{n}^{2}+6 \mu \varphi_{n} \mathcal{V}_{n}^{2}+9 \mu^{2} \varphi_{n}^{2} \mathcal{V}_{n}^{2}+6 \mu \varphi_{n} \mathcal{Z}_{n}-2 \mathcal{V}_{n} \mathcal{Z}_{n} \\
& -6 \mu \varphi_{n} \mathcal{V}_{n} \mathcal{Z}_{n}+\mathcal{Z}_{n}^{2} \\
= & \left(\mathcal{V}_{n}-3 \mu \varphi_{n}+3 \mu \varphi_{n} \mathcal{V}_{n}-\mathcal{Z}_{n}\right)^{2} .
\end{aligned}
$$

From (5.1), we have $G(y) \geq 0$, for every $y \in \mathbb{R}$. If $G(y)$ has at most one solution in $\mathbb{R}$, thus we have $\Delta \leq 0$. This implies that

$$
\mathcal{V}_{n}=\frac{\mathcal{Z}_{n}+3 \mu \varphi_{n}}{1+3 \mu \varphi_{n}}
$$

where $\mu=\sum_{i=1}^{N}\left(\frac{4}{5^{i}}+\frac{1}{N 5^{N}}\right) i$. Put $\delta_{n}=\frac{1}{3 n}, \theta_{n}=\frac{n}{2 n+3}, \varphi_{n}=\frac{2 n}{3 n+2}, \psi_{n}=\frac{1}{n^{2}}, \forall n \in \mathbb{N}$. It is clear to see that the sequences $\left\{\delta_{n}\right\},\left\{\theta_{n}\right\},\left\{\varphi_{n}\right\}$, and $\left\{\psi_{n}\right\}$ satisfy all conditions in Theorem 3.1. For every $n \in \mathbb{N}$, from (5.2), we rewrite (3.1) as follows:

$$
\left\{\begin{array}{l}
\mathcal{Y}_{n}=\frac{n}{2 n+3} P_{[1,100]} \mathcal{Z}_{n}+\left(1-\frac{n}{2 n+3}\right) \frac{1}{1+3 \mu \frac{2 n}{3 n+2}}\left(\mathcal{Z}_{n}+3 \mu \frac{2 n}{3 n+2}\right), \\
\mathcal{Z}_{n+1}=\frac{1}{36 n} \mathcal{Z}_{n}+\left(I-\frac{1}{3 n} \mathcal{A}\right) P_{[1,100]}\left(I-\frac{1}{n^{2}}(I-\mathcal{T})\right) \mathcal{Y}_{n}, \quad \forall n \in \mathbb{N} .
\end{array}\right.
$$

From Theorem 3.1, we can conclude that the sequences $\left\{\mathcal{Z}_{n}\right\},\left\{\mathcal{y}_{n}\right\}$, and $\left\{\mathcal{V}_{n}\right\}$ generated by (5.3) converge strongly to 1.

For case (i), with $N=1$, we have $\mu=1$. Then (5.2) becomes

$$
\mathcal{V}_{n}=\frac{\mathcal{Z}_{n}+3 \varphi_{n}}{1+3 \varphi_{n}} .
$$


Table 1 The values of $\left\{\mathcal{V}_{n}\right\},\left\{\mathscr{Y}_{n}\right\}$, and $\left\{\mathscr{Z}_{n}\right\}$ with $\mathscr{Z}_{1}=\mathbf{5 0}$

\begin{tabular}{|c|c|c|c|c|c|c|}
\hline \multirow[t]{2}{*}{$n$} & \multicolumn{3}{|l|}{$N=1$} & \multicolumn{3}{|l|}{$N=10$} \\
\hline & $\mathcal{V}_{n}$ & $y_{n}$ & $\mathcal{Z}_{n}$ & $\mathcal{V}_{n}$ & $y_{n}$ & $\mathfrak{Z}_{n}$ \\
\hline 1 & 23.272727 & 28.618182 & 50.000000 & 20.600006 & 26.480004 & 50.000000 \\
\hline 2 & 4.119192 & 5.455988 & 8.797980 & 3.535267 & 4.893445 & 8.288890 \\
\hline 3 & 2.287423 & 2.989653 & 4.394114 & 1.973077 & 2.636539 & 3.963461 \\
\hline 4 & 1.631883 & 2.025784 & 2.715111 & 1.446758 & 1.794880 & 2.404095 \\
\hline 5 & 1.331444 & 1.556406 & 1.916346 & 1.219216 & 1.405203 & 1.702781 \\
\hline 6 & 1.178502 & 1.307023 & 1.499805 & 1.109733 & 1.208492 & 1.356631 \\
\hline 7 & 1.096153 & 1.168453 & 1.271738 & 1.053976 & 1.104708 & 1.177182 \\
\hline 8 & 1.050342 & 1.089475 & 1.143282 & 1.024666 & 1.048632 & 1.081587 \\
\hline 9 & 1.024377 & 1.043831 & 1.069769 & 1.009019 & 1.018015 & 1.030010 \\
\hline 10 & 1.009539 & 1.017314 & 1.027423 & 1.000654 & 1.001320 & 1.002186 \\
\hline
\end{tabular}

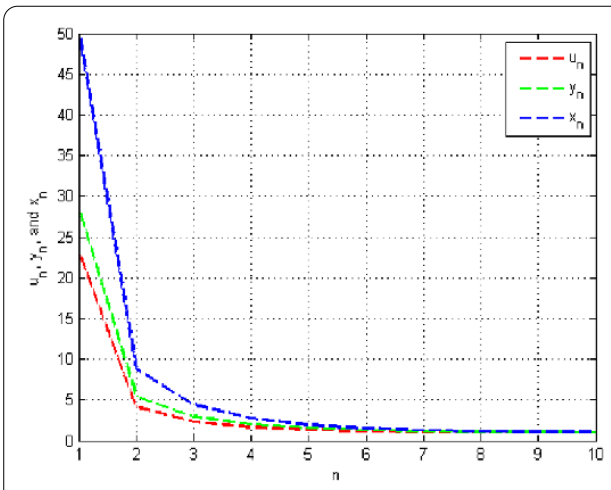

(a) $N=1$

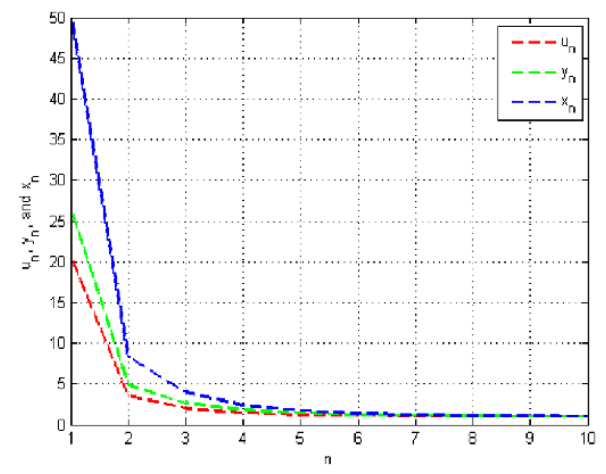

(b) $N=10$

Figure 1 The convergence of $\left\{\mathcal{V}_{n}\right\},\left\{\mathcal{Y}_{n}\right\}$, and $\left\{\mathcal{Z}_{n}\right\}$ with initial value $\mathscr{Z}_{1}=50$.

Then we have

$$
\left\{\begin{array}{l}
\mathcal{Y}_{n}=\frac{n}{2 n+3} P_{[1,100]} \mathcal{Z}_{n}+\left(1-\frac{n}{2 n+3}\right) \frac{1}{1+3 \frac{2 n}{3 n+2}}\left(\mathcal{Z}_{n}+3 \frac{2 n}{3 n+2}\right), \\
\mathcal{Z}_{n+1}=\frac{1}{36 n} \mathcal{Z}_{n}+\left(I-\frac{1}{3 n} \mathcal{A}\right) P_{[1,100]}\left(I-\frac{1}{n^{2}}(I-\mathcal{T})\right) \mathcal{Y}_{n}, \quad \forall n \in \mathbb{N}
\end{array}\right.
$$

From Corollary 3.2, we can conclude that the sequences $\left\{\mathcal{Z}_{n}\right\},\left\{\mathcal{Y}_{n}\right\}$, and $\left\{\mathcal{V}_{n}\right\}$ generated by (5.5) converge strongly to 1 .

Table 1 and Figure 1 show the values of sequences $\left\{\mathcal{Z}_{n}\right\},\left\{\mathcal{Y}_{n}\right\}$, and $\left\{\mathcal{V}_{n}\right\}$ in two cases.

\section{Remark 5.2}

(i) From Table 1 and Figure 1 , the sequences $\left\{\mathcal{Z}_{n}\right\},\left\{\mathcal{y}_{n}\right\}$, and $\left\{\mathcal{V}_{n}\right\}$ converge to 1 , where $\{1\}=\operatorname{Fix}(\mathcal{T}) \cap \bigcap_{i=1}^{N} \operatorname{EP}\left(\Phi_{i}\right)$.

(ii) For case (i), Corollary 3.2 guarantees the convergence of $\left\{\mathcal{Z}_{n}\right\},\left\{\mathcal{Y}_{n}\right\}$, and $\left\{\mathcal{V}_{n}\right\}$.

(iii) For case (ii), the convergence of $\left\{\mathcal{Z}_{n}\right\},\left\{\mathcal{Y}_{n}\right\}$, and $\left\{\mathcal{V}_{n}\right\}$ can be guaranteed by Theorem 3.1.

(iv) The iteration (5.3) for the combination of equilibrium problem converges faster than the iteration (5.5) for the classical equilibrium problem.

Finally, we give the numerical example for our main theorem in two-dimensional space of real numbers. 
Example 5.3 Let $\mathbb{R}^{2}$ be the two-dimensional space of real numbers with an inner product $\langle\cdot, \cdot\rangle: \mathbb{R}^{2} \times \mathbb{R}^{2} \rightarrow \mathbb{R}$ defined by $\langle u, v\rangle=u \cdot v=u_{1} v_{1}+u_{2} v_{2}$ and a usual norm $\|\cdot\|: \mathbb{R}^{2} \rightarrow \mathbb{R}$ given by $\|u\|=\sqrt{u_{1}^{2}+u_{2}^{2}}$, for all $u=\left(u_{1}, u_{2}\right), v=\left(v_{1}, v_{2}\right) \in \mathbb{R}^{2}$. Let the mappings $\mathcal{A}: \mathbb{R}^{2} \rightarrow$ $\mathbb{R}^{2}, \mathcal{F}: \mathbb{R}^{2} \rightarrow \mathbb{R}^{2}$ be defined by

$$
\begin{aligned}
& \mathcal{A} u=\left(\frac{u_{1}}{2}, \frac{u_{2}}{2}\right), \\
& \mathcal{F} u=\left(\frac{u_{1}}{4}, \frac{u_{2}}{4}\right), \quad \text { for all } u=\left(u_{1}, u_{2}\right) \in \mathbb{R}^{2} .
\end{aligned}
$$

For every $i=1,2, \ldots, N$ and $I=[0,1]$, let $\Phi_{i}: I^{2} \times I^{2} \rightarrow \mathbb{R}$ and $\mathcal{T}: I^{2} \rightarrow I^{2}$ be defined by

$$
\begin{aligned}
& \mathcal{T} u=\left(\frac{u_{1}+1}{2}, \frac{5 u_{2}+1}{6}\right), \\
& \Phi_{i}(u, v)=i(v-u) \cdot(v+6 u-7), \quad \text { for all } u=\left(u_{1}, u_{2}\right), v=\left(v_{1}, v_{2}\right) \in I^{2},
\end{aligned}
$$

where $7=(7,7)$. Let $\gamma=\frac{1}{3}, \delta_{n}=\frac{1}{3 n}, \theta_{n}=\frac{n}{2 n+3}, \varphi_{n}=\frac{2 n}{3 n+2}$, and $\psi_{n}=\frac{1}{n^{2}}$ for every $n \in \mathbb{N}$.

It is clear that $\mathcal{T}$ is a nonspreading mapping and $\operatorname{Fix}(\mathcal{T})=\{\mathbf{1}\}$, where $\mathbf{1}=(1,1)$.

Put $a_{i}=\frac{8}{9^{i}}+\frac{1}{N 9^{N}}$, for every $i=1,2, \ldots, N$. It is obvious that $\sum_{i=1}^{N} a_{i} \Phi_{i}$ satisfies all conditions in Theorem 3.1 and $\operatorname{EP}\left(\sum_{i=1}^{N} a_{i} \Phi_{i}\right)=\bigcap_{i=1}^{N} \operatorname{EP}\left(\Phi_{i}\right)=\{\mathbf{1}\}$, where $\mathbf{1}=(1,1)$. Then we have

$$
\operatorname{Fix}(\mathcal{T}) \cap \bigcap_{i=1}^{N} \operatorname{EP}\left(\Phi_{i}\right)=\{\mathbf{1}\} .
$$

Then, by Theorem 3.1, the sequences $\mathcal{Z}_{n}=\left(\mathcal{Z}_{n}^{1}, \mathcal{Z}_{n}^{2}\right), \mathcal{Y}_{n}=\left(\mathcal{Y}_{n}^{1}, \mathcal{Y}_{n}^{2}\right)$, and $\mathcal{V}_{n}=\left(\mathcal{V}_{n}^{1}, \mathcal{V}_{n}^{2}\right)$ converge strongly to $\{\mathbf{1}\}$.

Remark 5.4 From Example 5.3, putting $\rho=\sum_{i=1}^{N}\left(\frac{8}{9^{i}}+\frac{1}{N 9^{N}}\right) i$, we obtain

$$
\begin{aligned}
0 \leq & \sum_{i=1}^{N} a_{i} \Phi_{i}\left(\mathcal{V}_{n}, y\right)+\frac{1}{\varphi_{n}}\left\langle y-\mathcal{V}_{n}, \mathcal{V}_{n}-\mathcal{Z}_{n}\right\rangle \\
= & \rho\left(y-\mathcal{V}_{n}\right) \cdot\left(y+6 \mathcal{V}_{n}-(7,7)\right)+\frac{1}{\varphi_{n}}\left(y-\mathcal{V}_{n}\right) \cdot\left(\mathcal{V}_{n}-\mathcal{Z}_{n}\right) \\
= & \rho\left(y_{1}-\mathcal{V}_{n}^{1}, y_{2}-\mathcal{V}_{n}^{2}\right) \cdot\left(y_{1}+6 \mathcal{V}_{n}^{1}-7, y_{2}+6 \mathcal{V}_{n}^{2}-7\right) \\
& +\frac{1}{\varphi_{n}}\left(y_{1}-\mathcal{V}_{n}^{1}, y_{2}-\mathcal{V}_{n}^{2}\right) \cdot\left(\mathcal{V}_{n}^{1}-\mathcal{Z}_{n}^{1}, \mathcal{V}_{n}^{2}-\mathcal{Z}_{n}^{2}\right) \\
= & \rho\left(\left(y_{1}-\mathcal{V}_{n}^{1}\right)\left(y_{1}+6 \mathcal{V}_{n}^{1}-7\right)+\left(y_{2}-\mathcal{V}_{n}^{2}\right)\left(y_{2}+6 \mathcal{V}_{n}^{2}-7\right)\right) \\
& +\frac{1}{\varphi_{n}}\left(\left(y_{1}-\mathcal{V}_{n}^{1}\right)\left(\mathcal{V}_{n}^{1}-\mathcal{Z}_{n}^{1}\right)+\left(y_{2}-\mathcal{V}_{n}^{2}\right)\left(\mathcal{V}_{n}^{2}-\mathcal{Z}_{n}^{2}\right)\right) \\
= & \left(\rho\left(y_{1}-\mathcal{V}_{n}^{1}\right)\left(y_{1}+6 \mathcal{V}_{n}^{1}-7\right)+\frac{1}{\varphi_{n}}\left(y_{1}-\mathcal{V}_{n}^{1}\right)\left(\mathcal{V}_{n}^{1}-\mathcal{Z}_{n}^{1}\right)\right) \\
& +\left(\rho\left(y_{2}-\mathcal{V}_{n}^{2}\right)\left(y_{2}+6 \mathcal{V}_{n}^{2}-7\right)+\frac{1}{\varphi_{n}}\left(y_{2}-\mathcal{V}_{n}^{2}\right)\left(\mathcal{V}_{n}^{2}-\mathcal{Z}_{n}^{2}\right)\right) \\
\Leftrightarrow & 0 \leq\left(\rho \varphi_{n}\left(y_{1}-\mathcal{V}_{n}^{1}\right)\left(y_{1}+6 \mathcal{V}_{n}^{1}-7\right)+\left(y_{1}-\mathcal{V}_{n}^{1}\right)\left(\mathcal{V}_{n}^{1}-\mathcal{Z}_{n}^{1}\right)\right)
\end{aligned}
$$




$$
\begin{aligned}
& +\left(\rho \varphi_{n}\left(y_{2}-\mathcal{V}_{n}^{2}\right)\left(y_{2}+6 \mathcal{V}_{n}^{2}-7\right)+\left(y_{2}-\mathcal{V}_{n}^{2}\right)\left(\mathcal{V}_{n}^{2}-\mathcal{Z}_{n}^{2}\right)\right) \\
= & \left(\rho \varphi_{n}\left(y_{1}\right)^{2}+\left(5 \rho \mathcal{V}_{n}^{1} \varphi_{n}+\mathcal{V}_{n}^{1}-\mathcal{Z}_{n}^{1}-7 \rho \varphi_{n}\right) y_{1}\right. \\
& \left.+7 \rho \varphi_{n} \mathcal{V}_{n}^{1}-\left(\mathcal{V}_{n}^{1}\right)^{2}-6 \rho \varphi_{n}\left(\mathcal{V}_{n}^{1}\right)^{2}+\mathcal{V}_{n}^{1} \mathcal{Z}_{n}^{1}\right) \\
& +\left(\rho \varphi_{n}\left(y_{2}\right)^{2}+\left(5 \rho \mathcal{V}_{n}^{2} \varphi_{n}+\mathcal{V}_{n}^{2}-\mathcal{Z}_{n}^{2}-7 \rho \varphi_{n}\right) y_{2}\right. \\
& \left.+7 \rho \varphi_{n} \mathcal{V}_{n}^{2}-\left(\mathcal{V}_{n}^{2}\right)^{2}-6 \rho \varphi_{n}\left(\mathcal{V}_{n}^{2}\right)^{2}+\mathcal{V}_{n}^{2} \mathcal{Z}_{n}^{2}\right) \\
= & G_{1}\left(y_{1}\right)+G_{2}\left(y_{2}\right),
\end{aligned}
$$

where $G_{1}\left(y_{1}\right)=\rho \varphi_{n}\left(y_{1}\right)^{2}+\left(5 \rho \mathcal{V}_{n}^{1} \varphi_{n}+\mathcal{V}_{n}^{1}-\mathcal{Z}_{n}^{1}-7 \rho \varphi_{n}\right) y_{1}+7 \rho \varphi_{n} \mathcal{V}_{n}^{1}-\left(\mathcal{V}_{n}^{1}\right)^{2}-6 \rho \varphi_{n}\left(\mathcal{V}_{n}^{1}\right)^{2}+$ $\mathcal{V}_{n}^{1} \mathcal{Z}_{n}^{1}$ and $G_{2}\left(y_{2}\right)=\rho \varphi_{n}\left(y_{2}\right)^{2}+\left(5 \rho \mathcal{V}_{n}^{2} \varphi_{n}+\mathcal{V}_{n}^{2}-\mathcal{Z}_{n}^{2}-7 \rho \varphi_{n}\right) y_{2}+7 \rho \varphi_{n} \mathcal{V}_{n}^{2}-\left(\mathcal{V}_{n}^{2}\right)^{2}-6 \rho \varphi_{n}\left(\mathcal{V}_{n}^{2}\right)^{2}+$ $\mathcal{V}_{n}^{2} \mathcal{Z}_{n}^{2}$. Then $G_{1}\left(y_{1}\right)$ and $G_{2}\left(y_{2}\right)$ are quadratic functions with coefficients $a_{1}=\rho \varphi_{n}, b_{1}=$ $5 \rho \mathcal{V}_{n}^{1} \varphi_{n}+\mathcal{V}_{n}^{1}-\mathcal{Z}_{n}^{1}-7 \rho \varphi_{n}$, and $c_{1}=7 \rho \varphi_{n} \mathcal{V}_{n}^{1}-\left(\mathcal{V}_{n}^{1}\right)^{2}-6 \rho \varphi_{n}\left(\mathcal{V}_{n}^{1}\right)^{2}+\mathcal{V}_{n}^{1} \mathcal{Z}_{n}^{1}$, and $a_{2}=\rho \varphi_{n}$, $b_{2}=5 \rho \mathcal{V}_{n}^{2} \varphi_{n}+\mathcal{V}_{n}^{2}-\mathcal{Z}_{n}^{2}-7 \rho \varphi_{n}$, and $c_{2}=7 \rho \varphi_{n} \mathcal{V}_{n}^{2}-\left(\mathcal{V}_{n}^{2}\right)^{2}-6 \rho \varphi_{n}\left(\mathcal{V}_{n}^{2}\right)^{2}+\mathcal{V}_{n}^{2} \mathcal{Z}_{n}^{2}$, respectively.

Determine the discriminant $\Delta_{1}$ of $G_{1}$ as follows:

$$
\begin{aligned}
\Delta_{1} & =\left(b_{1}\right)^{2}-4 a_{1} c_{1} \\
& =\left(5 \rho \mathcal{V}_{n}^{1} \varphi_{n}+\mathcal{V}_{n}^{1}-\mathcal{Z}_{n}^{1}-7 \rho \varphi_{n}\right)^{2}-4 \rho \varphi_{n}\left(7 \rho \varphi_{n} \mathcal{V}_{n}^{1}-\left(\mathcal{V}_{n}^{1}\right)^{2}-6 \rho \varphi_{n}\left(\mathcal{V}_{n}^{1}\right)^{2}+\mathcal{V}_{n}^{1} \mathcal{Z}_{n}^{1}\right) \\
& =\left(\mathcal{V}_{n}^{1}-7 \rho \varphi_{n}+7 \rho \varphi_{n} \mathcal{V}_{n}^{1}-\mathcal{Z}_{n}^{1}\right)^{2} .
\end{aligned}
$$

From (5.6), if $G_{1}\left(y_{1}\right) \geq 0, \forall y_{1} \in \mathbb{R}$, it has at most one solution in $\mathbb{R}$, thus $\Delta_{1} \leq 0$. It follows that

$$
\mathcal{V}_{n}^{1}=\frac{\mathcal{Z}_{n}^{1}+7 \rho \varphi_{n}}{1+7 \rho \varphi_{n}}
$$

Next, we determine the discriminant $\Delta_{2}$ of $G_{2}$ as follows:

$$
\begin{aligned}
\Delta_{2} & =\left(b_{2}\right)^{2}-4 a_{2} c_{2} \\
& =\left(5 \rho \mathcal{V}_{n}^{2} \varphi_{n}+\mathcal{V}_{n}^{2}-\mathcal{Z}_{n}^{2}-7 \rho \varphi_{n}\right)^{2}-4 \rho \varphi_{n}\left(7 \rho \varphi_{n} \mathcal{V}_{n}^{2}-\left(\mathcal{V}_{n}^{2}\right)^{2}-6 \rho \varphi_{n}\left(\mathcal{V}_{n}^{2}\right)^{2}+\mathcal{V}_{n}^{2} \mathcal{Z}_{n}^{2}\right) \\
& =\left(\mathcal{V}_{n}^{2}-7 \rho \varphi_{n}+7 \rho \varphi_{n} \mathcal{V}_{n}^{2}-\mathcal{Z}_{n}^{2}\right)^{2} .
\end{aligned}
$$

From (5.6), if $G_{2}\left(y_{2}\right) \geq 0, \forall y_{2} \in \mathbb{R}$ and it has at most one solution in $\mathbb{R}$, then $\Delta_{2} \leq 0$. This yields

$$
\mathcal{V}_{n}^{2}=\frac{\mathcal{Z}_{n}^{2}+7 \rho \varphi_{n}}{1+7 \rho \varphi_{n}}
$$

Put $\delta_{n}=\frac{1}{3 n}, \theta_{n}=\frac{n}{2 n+3}, \varphi_{n}=\frac{2 n}{3 n+2}, \psi_{n}=\frac{1}{n^{2}}$, for all $n \in \mathbb{N}$. It is obvious that the sequences $\left\{\delta_{n}\right\}$, $\left\{\theta_{n}\right\},\left\{\varphi_{n}\right\}$, and $\left\{\psi_{n}\right\}$ satisfy all conditions in Theorem 3.1. For every $n \in \mathbb{N}$, from (5.7) and (5.8), the iterative scheme (3.1) becomes

$$
\left\{\begin{array}{l}
y_{n}=\frac{n}{2 n+3} P_{C} \mathcal{Z}_{n}+\left(1-\frac{n}{2 n+3}\right) \mathcal{U}_{n}, \\
\mathcal{Z}_{n+1}=\frac{1}{36 n} \mathcal{Z}_{n}+\left(I-\frac{1}{3 n} A\right) P_{C}\left(I-\frac{1}{n^{2}}(I-\mathcal{T})\right) \mathcal{Y}_{n}, \quad \forall n \in \mathbb{N},
\end{array}\right.
$$

where $\mathcal{Z}_{n}=\left(\mathcal{Z}_{n}^{1}, \mathcal{Z}_{n}^{2}\right), \mathcal{Y}_{n}=\left(\mathcal{Y}_{n}^{1}, \mathcal{Y}_{n}^{2}\right)$, and $\mathcal{V}_{n}=\left(\mathcal{V}_{n}^{1}, \mathcal{V}_{n}^{2}\right)=\left(\frac{\mathcal{Z}_{n}^{1}+7 \rho \varphi_{n}}{1+7 \rho \varphi_{n}}, \frac{\mathcal{Z}_{n}^{2}+7 \rho \varphi_{n}}{1+7 \rho \varphi_{n}}\right)$ 
Let the initial values be defined as in the following cases.

(i) $Z_{1}=\left(Z_{1}^{1}, Z_{1}^{2}\right)=(-1,0), N=1$, and $n=20$,

(ii) $Z_{1}=\left(Z_{1}^{1}, Z_{1}^{2}\right)=(-1,0)$ and $n=N=20$.

For case (i), with $N=1$, we have $\rho=1$. Then, from (5.7) and (5.8), we obtain

$$
\mathcal{V}_{n}^{1}=\frac{\mathcal{Z}_{n}^{1}+3 \varphi_{n}}{1+7 \varphi_{n}}
$$

and

$$
\mathcal{V}_{n}^{2}=\frac{\mathcal{Z}_{n}^{2}+3 \varphi_{n}}{1+7 \varphi_{n}}
$$

Then we have

$$
\left\{\begin{array}{l}
y_{n}=\frac{n}{2 n+3} P_{C} \mathcal{Z}_{n}+\left(1-\frac{n}{2 n+3}\right) \mathcal{V}_{n}, \\
\mathcal{Z}_{n+1}=\frac{1}{36 n} \mathcal{Z}_{n}+\left(I-\frac{1}{3 n} A\right) P_{C}\left(I-\frac{1}{n^{2}}(I-\mathcal{T})\right) \mathcal{Y}_{n}, \quad \forall n \in \mathbb{N},
\end{array}\right.
$$

where $\mathcal{Z}_{n}=\left(\mathcal{Z}_{n}^{1}, \mathcal{Z}_{n}^{2}\right), \mathcal{Y}_{n}=\left(\mathcal{Y}_{n}^{1}, y_{n}^{2}\right)$, and $\mathcal{V}_{n}=\left(\mathcal{V}_{n}^{1}, \mathcal{V}_{n}^{2}\right)=\left(\frac{\mathcal{Z}_{n}^{1}+3 \varphi_{n}}{1+7 \varphi_{n}}, \frac{\mathcal{Z}_{n}^{2}+3 \varphi_{n}}{1+7 \varphi_{n}}\right)$.

Tables 2 and 3 and Figure 2 show the values of the sequences $\left\{\mathcal{Z}_{n}\right\},\left\{\mathcal{Y}_{n}\right\}$, and $\left\{\mathcal{V}_{n}\right\}$ in the two cases.

Table 2 The values of $\left\{\mathcal{V}_{n}\right\},\left\{\mathcal{Y}_{n}\right\}$, and $\left\{\mathcal{Z}_{n}\right\}$ with $\mathscr{Z}_{1}=(-1,0)$ and $N=1$

\begin{tabular}{rlll}
\hline $\boldsymbol{n}$ & $\boldsymbol{V}_{\boldsymbol{n}}=\left(\mathcal{V}_{\boldsymbol{n}}^{\mathbf{1}}, \boldsymbol{V}_{\boldsymbol{n}}^{\mathbf{2}}\right)$ & $\boldsymbol{Y}_{\boldsymbol{n}}=\left(\boldsymbol{Y}_{\boldsymbol{n}}^{\mathbf{1}}, \boldsymbol{Y}_{\boldsymbol{n}}^{\mathbf{2}}\right)$ & $\boldsymbol{Z}_{\boldsymbol{n}}=\left(\mathcal{Z}_{\boldsymbol{n}}^{\mathbf{1}}, \boldsymbol{Z}_{\boldsymbol{n}}^{\mathbf{2}}\right)$ \\
\hline 1 & $(0.473684,0.736842)$ & $(0.178947,0.589474)$ & $(-1.000000,0.000000)$ \\
2 & $(0.880767,0.899610)$ & $(0.761533,0.799220)$ & $(0.463450,0.548246)$ \\
3 & $(0.944343,0.947678)$ & $(0.873506,0.881086)$ & $(0.731833,0.747902)$ \\
4 & $(0.967678,0.968228)$ & $(0.920665,0.922015)$ & $(0.838391,0.841141)$ \\
5 & $(0.978604,0.978548)$ & $(0.944718,0.944575)$ & $(0.890502,0.890217)$ \\
$\vdots$ & $\vdots$ & $\vdots$ & $\vdots$ \\
10 & $(0.992787,0.992723)$ & $(0.979067,0.978881)$ & $(0.961231,0.960887)$ \\
$\vdots$ & $\vdots$ & $\vdots$ & $\vdots$ \\
16 & $(0.995890,0.995878)$ & $(0.987473,0.987437)$ & $(0.977477,0.977412)$ \\
17 & $(0.996158,0.996149)$ & $(0.988232,0.988203)$ & $(0.978908,0.978856)$ \\
18 & $(0.996393,0.996386)$ & $(0.988903,0.988879)$ & $(0.980164,0.980121)$ \\
19 & $(0.996601,0.996595)$ & $(0.989499,0.989479)$ & $(0.981276,0.981241)$ \\
20 & $(0.996785,0.996780)$ & $(0.990033,0.990017)$ & $(0.982268,0.982239)$ \\
\hline
\end{tabular}

Table 3 The values of $\left\{\mathcal{V}_{n}\right\},\left\{\mathcal{Y}_{n}\right\}$, and $\left\{\mathcal{Z}_{n}\right\}$ with $\mathscr{Z}_{1}=(-1,0), N=20$

\begin{tabular}{rlll}
\hline $\boldsymbol{n}$ & $\mathcal{V}_{\boldsymbol{n}}=\left(\mathcal{V}_{\boldsymbol{n}}^{\mathbf{1}}, \boldsymbol{V}_{\boldsymbol{n}}^{\mathbf{2}}\right)$ & $\boldsymbol{Y}_{\boldsymbol{n}}=\left(\boldsymbol{y}_{\boldsymbol{n}}^{\mathbf{1}}, \boldsymbol{Y}_{\boldsymbol{n}}^{\mathbf{2}}\right)$ & $\mathfrak{Z}_{\boldsymbol{n}}=\left(\mathcal{Z}_{\boldsymbol{n}}^{\mathbf{1}}, \mathfrak{Z}_{\boldsymbol{n}}^{\mathbf{2}}\right)$ \\
\hline 1 & $(0.518072,0.759036)$ & $(0.214458,0.607229)$ & $(-1.000000,0.000000)$ \\
2 & $(0.894328,0.911003)$ & $(0.775448,0.810881)$ & $(0.478246,0.560576)$ \\
3 & $(0.951505,0.954360)$ & $(0.882070,0.889012)$ & $(0.743199,0.758316)$ \\
4 & $(0.972025,0.972470)$ & $(0.926247,0.927421)$ & $(0.846135,0.848585)$ \\
5 & $(0.981489,0.981428)$ & $(0.948508,0.948339)$ & $(0.895738,0.895396)$ \\
$\vdots$ & $\vdots$ & $\vdots$ & $\vdots$ \\
10 & $(0.993655,0.993602)$ & $(0.980077,0.979909)$ & $(0.962426,0.962110)$ \\
$\vdots$ & $\vdots$ & $\vdots$ & $\vdots$ \\
16 & $(0.996360,0.996351)$ & $(0.987975,0.987942)$ & $(0.978017,0.977957)$ \\
17 & $(0.996597,0.996589)$ & $(0.988698,0.988671)$ & $(0.979405,0.979357)$ \\
18 & $(0.996804,0.996798)$ & $(0.989336,0.989315)$ & $(0.980624,0.980585)$ \\
19 & $(0.996987,0.996982)$ & $(0.989905,0.989888)$ & $(0.981706,0.981673)$ \\
20 & $(0.997150,0.997146)$ & $(0.990416,0.990401)$ & $(0.982671,0.982644)$ \\
\hline
\end{tabular}




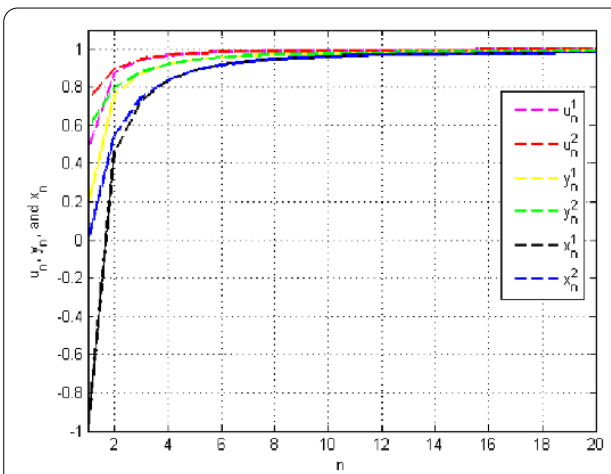

(a) $N=1$

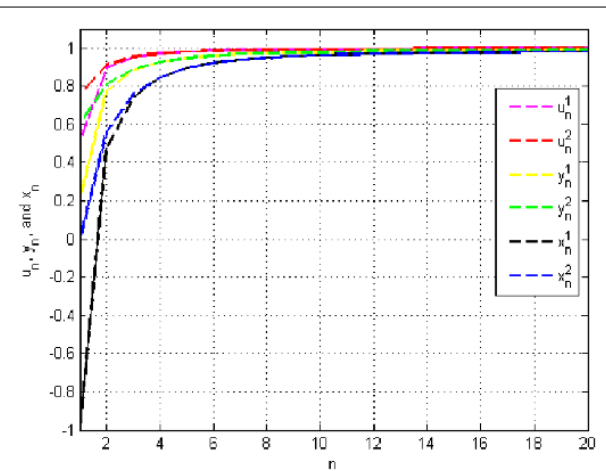

(b) $N=10$

Figure 2 The convergence of $\left\{\mathcal{V}_{n}\right\},\left\{\mathcal{Y}_{n}\right\}$, and $\left\{\mathcal{L}_{n}\right\}$ with initial value $\mathscr{Z}_{1}=(-1,0)$ for both cases.

\section{Remark 5.5}

(i) Tables 2 and 3 , and Figure 2 show that the sequences $\left\{\mathcal{Z}_{n}\right\},\left\{\mathcal{Y}_{n}\right\}$, and $\left\{\mathcal{V}_{n}\right\}$ converge to $\mathbf{1}$, where $\{\mathbf{1}\}=\{(1,1)\}=\operatorname{Fix}(\mathcal{T}) \cap \bigcap_{i=1}^{N} \operatorname{EP}\left(\Phi_{i}\right)$.

(ii) For case (i), Corollary 3.2 guarantees the convergence of $\left\{\mathcal{Z}_{n}\right\},\left\{\mathcal{Y}_{n}\right\}$, and $\left\{\mathcal{V}_{n}\right\}$.

(iii) For case (ii), the convergence of $\left\{\mathcal{Z}_{n}\right\},\left\{\mathcal{y}_{n}\right\}$, and $\left\{\mathcal{V}_{n}\right\}$ can be guaranteed by Theorem 3.1.

(iv) The iteration (5.9) for the combination of equilibrium problem converges faster than the iteration (5.10) for the classical equilibrium problem.

\section{Competing interests}

The authors declare that they have no competing interests.

\section{Authors' contributions}

Both authors contributed equally and significantly to this research article. Both authors read and approved the final manuscript.

\section{Acknowledgements}

The authors appreciated the referees providing valuable comments improving the content of this research paper. This research is supported by the Research Administration Division of King Mongkut's Institute of Technology Ladkrabang.

Received: 31 January 2014 Accepted: 19 July 2014 Published: 15 Aug 2014

\section{References}

1. Kohsaka, F, Takahashi, W: Fixed point theorems for a class of nonlinear mappings related to maximal monotone operators in Banach spaces. Arch. Math. 91, 166-177 (2008)

2. lemoto, S, Takahashi, W: Approximating common fixed points of nonexpansive mappings and nonspreading mappings in a Hilbert space. Nonlinear Anal. 71, 2082-2089 (2009)

3. Kurokawa, Y, Takahashi, W: Weak and strong convergence theorems for nonspreading mappings in Hilbert spaces. Nonlinear Anal. 73, 1562-1568 (2010)

4. Osilike, MO, Isiogugu, FO: Weak and strong convergence theorems for nonspreading-type mappings in Hilbert spaces. Nonlinear Anal. 74, 1814-1822 (2011)

5. Liu, H, Wang, J, Feng, Q: Strong convergence theorems for maximal monotone operators with nonspreading mappings in a Hilbert space. Abstr. Appl. Anal. (2012). doi:10.1155/2012/917857

6. Deng, BC, Chen, T, Li, FL: Viscosity iteration algorithm for a $\rho$-strictly pseudononspreading mapping in a Hilbert space. J. Inequal. Appl. 2013, 80 (2013)

7. Blum, E, Oettli, W: From optimization and variational inequalities to equilibrium problems. Math. Stud. 63(14), 123-145 (1994)

8. Combettes, PL, Hirstoaga, SA: Equilibrium programming in Hilbert spaces. J. Nonlinear Convex Anal. 6(1), 117-136 (2005)

9. Ceng, LC, Al-Homidan, S, Ansari, QH, Yao, JC: An iterative scheme for equilibrium problems and fixed point problems of strict pseudo-contraction mappings. J. Comput. Appl. Math. 223, 967-974 (2009)

10. Ceng, LC, Ansari, QH, Yao, JC: Viscosity approximation methods for generalized equilibrium problems and fixed point problems. J. Glob. Optim. 43, 487-502 (2009). doi:10.1007/s10898-008-9342-6 
11. Ceng, LC, Ansari, QH, Yao, JC: Hybrid pseudoviscosity approximation schemes for equilibrium problems and fixed point problems of infinitely many nonexpansive mappings. Nonlinear Anal. Hybrid Syst. 4, 743-754 (2010)

12. Suwannaut, S, Kangtunyakarn, A: The combination of the set of solutions of equilibrium problem for convergence theorem of the set of fixed points of strictly pseudo-contractive mappings and variational inequalities problem. Fixed Point Theory Appl. 2013, 291 (2013)

13. Takahashi, W, Shimoji, K: Convergence theorems for nonexpansive mappings and feasibility problems. Math. Comput. Model. 32, 1463-1471 (2000)

14. Takahashi, S, Takahashi, W: Viscosity approximation methods for equilibrium problems and fixed point problems in Hilbert spaces. J. Math. Anal. Appl. 331(1), 506-515 (2007)

15. Peng, JW, Wu, SY, Yao, JC: A new iterative method for finding common solutions of a system of equilibrium problems, fixed-point problems, and variational inequalities. Abstr. Appl. Anal. (2010). doi:10.1155/2010/428293

16. Saeidi, S: Iterative algorithms for finding common solutions of variational inequalities and systems of equilibrium problems and fixed points of families and semigroups of nonexpansive mappings. Nonlinear Anal. 70, 4195-4208 (2009)

17. Piri, $\mathrm{H}$ : A general iterative method for finding common solutions of system of equilibrium problems, system of variational inequalities and fixed point problems. Math. Comput. Model. 55, 1622-1638 (2012)

18. Jitpeera, T, Kumam, P: An extragradient type method for a system of equilibrium problems, variational inequality problems and fixed points of finitely many nonexpansive mappings. J. Nonlinear Anal. Optim. 1(1), 71-91 (2010)

19. Plubtieng, S, Punpaeng, R: A general iterative method for equilibrium problems and fixed point problems in Hilbert spaces. J. Math. Anal. Appl. 336(1), 455-469 (2007)

20. Takahashi, W: Nonlinear Functional Analysis. Yokohama Publishers, Yokohama (2000)

21. Opial, Z: Weak convergence of the sequence of successive approximation of nonexpansive mappings. Bull. Am. Math. Soc. 73, 591-597 (1967)

22. Xu, HK: An iterative approach to quadric optimization. J. Optim. Theory Appl. 116, 659-678 (2003)

23. Marino, G, Xu, HK: A general iterative method for nonexpansive mappings in Hilbert spaces. J. Math. Anal. Appl. 318 43-52 (2006)

24. Kangtunyakarn, A, Suantai, S: Hybrid iterative scheme for generalized equilibrium problems and fixed point problems of finite family of nonexpansive mappings. Nonlinear Anal. Hybrid Syst. 3, 296-309 (2009)

25. Kangtunyakarn, A: Strong convergence of the hybrid method for a finite family of nonspreading mappings and variational inequality problems. Fixed Point Theory Appl. 2012, 188 (2012)

26. Tian, $M$, Jin, X: Strong convergence result for quasi-nonexpansive mappings in Hilbert spaces. Fixed Point Theory Appl. 2011, 88 (2011). doi:10.1186/1687-1812-2011-88

10.1186/1687-1812-2014-167

Cite this article as: Suwannaut and Kangtunyakarn: Convergence analysis for the equilibrium problems with numerical results. Fixed Point Theory and Applications 2014, 2014:167

\section{Submit your manuscript to a SpringerOpen ${ }^{\circ}$ journal and benefit from:}

- Convenient online submission

- Rigorous peer review

- Immediate publication on acceptance

- Open access: articles freely available online

- High visibility within the field

- Retaining the copyright to your article 